\title{
Dynamics of Lower-Tropospheric Vorticity in Idealized Simulations of Tropical Cyclone Formation
}

\author{
YAPING WANG, Christopher A. DAvis, AND YongJie HuANG \\ National Center for Atmospheric Research, Boulder, Colorado
}

(Manuscript received 31 July 2018, in final form 30 December 2018)

\begin{abstract}
Idealized simulations are conducted using the Cloud Model version 1 (CM1) to explore the mechanism of tropical cyclone (TC) genesis from a preexisting midtropospheric vortex that forms in radiative-convective equilibrium. With lower-tropospheric air approaching near saturation during TC genesis, convective cells become stronger, along with the intensifying updrafts and downdrafts and the larger area coverage of updrafts relative to downdrafts. Consequently, the low-level vertical mass flux increases, inducing vorticity amplification above the boundary layer. Of interest is that while surface cold pools help organize lowertropospheric updrafts, genesis still proceeds, only slightly delayed, if subcloud evaporation cooling and cold pool intensity are drastically reduced. More detrimental is the disruption of near saturation through the introduction of weak vertical wind shear. The lower-tropospheric dry air suppresses the strengthening of convection, leading to weaker upward mass flux and much slower near-surface vortex spinup. We also find that surface spinup is similarly inhibited by decreasing surface drag despite the existence of a nearly saturated column, whereas larger drag accelerates spinup. Increased vorticity above the boundary layer is followed by the emergence of a horizontal pressure gradient through the depth of the boundary layer. Then the corresponding convergence resulting from the gradient imbalance in the frictional boundary layer causes vorticity amplification near the surface. It is suggested that near saturation in the lower troposphere is critical for increasing the mass flux and vorticity just above the boundary layer, but it is necessary yet insufficient because the spinup is strongly governed by boundary layer dynamics.
\end{abstract}

\section{Introduction}

The tropical cyclone (TC) genesis phase is one aspect of the TC life cycle lacking sufficient understanding. The development of strong near-surface cyclonic vorticity is a key element of TC spinup, and different ideas have been introduced to describe how this process occurs. One idea, articulated in both numerical simulations (Raymond and Sessions 2007) and observation analyses (Raymond and López-Carrillo 2011; Gjorgjievska and Raymond 2014), is that antecedent midtropospheric cyclonic vorticity aids the spinup of a vortex in the boundary layer through a strong increase of the vertical mass flux with height at low levels and a corresponding horizontal convergence of mass. In this process, a moist, midlevel vortex, which is considered as the precursor of TC genesis by several studies (Ritchie and Holland 1997; Simpson et al. 1997; Bister and Emanuel 1997), is suggested to be conducive to convection and favorable for the transition

Corresponding author: Yaping Wang,wangyap1990@gmail.com of mass flux profile from top-heavy to bottom-heavy (Raymond et al. 2011; Raymond 2012). As a result, the increasing vertical gradient of vertical mass flux at low levels induces near-surface convergence.

Hendricks et al. (2004) pointed out that those rotating updrafts, called vortical hot towers (VHTs), rotate rapidly enough to suppress entrainment and, thus, alter the vertical mass flux profile in tropical cyclogenesis, although these updrafts do not need to penetrate all the way to the tropopause. Recently, a series of papers (Nguyen et al. 2008; Smith et al. 2009; Montgomery et al. 2009; Persing et al. 2013) proposed a rotating convective updraft paradigm for vortex intensification, in which the development, merger, and axisymmetrization of cyclonically rotating updrafts cause the upscale growth of cyclonic vorticity. This VHT paradigm works similarly in tropical cyclogenesis and TC intensification, though the pattern of deep convection and the associated cyclonic vorticity during genesis process may be far from axisymmetric (Kilroy et al. 2017a). The existence of VHTs is validated by observations (Reasor et al. 2005; Sippel et al. 2006; 
Houze et al. 2009; Bell and Montgomery 2010; Sanger et al. 2014). As convection develops in tropical cyclogenesis, vorticity is amplified by stretching of ambient vortex vorticity and tilting of horizontal vorticity into the vertical by convective updrafts and downdrafts (Montgomery et al. 2006). The amplified cyclonic vorticity, which outlasts the updrafts themselves, grows horizontally in scale due to merger and axisymmetrization with adjacent vorticity anomalies (Montgomery and Smith 2014).

Smith et al. (2009) identified two mechanisms for the spinup of the mean tangential circulation in $\mathrm{TC}$ intensification. The first mechanism is associated with the radial convergence of absolute angular momentum above the boundary layer induced by the inner-core convection, and the second mechanism involves the convergence of absolute angular momentum within the boundary layer, called the boundary layer spinup mechanism. The second mechanism is associated with radial convergence and is likely enhanced by the gradient wind imbalance within the boundary layer. The mechanism stresses the fact that the spinup of the maximum tangential winds occurs in the boundary layer. It requires the radial pressure gradient to increase and the spinup of tangential wind at the top of the boundary layer by the first mechanism. The boundary layer spinup mechanism is supported by numerical simulations of idealized configurations and real cases (Zhang et al. 2001; Smith et al. 2009; Abarca and Montgomery 2013; Persing et al. 2013; Zhang and Marks 2015; Schmidt and Smith 2016). Not unique to TC intensification, this mechanism is a feature of other rapidly rotating atmospheric vortices such as tornadoes, water spouts, and dust devils (Lewellen and Lewellen 2007; Montgomery and Smith 2014).

Many studies have shown that the thermodynamic environment plays an important role on the path to TC formation from precursor tropical disturbances. This paper seeks to understand the relative importance of the aforementioned ideas during the earliest stages of genesis and explores the effects of thermodynamics, such as air temperature and humidity, on convection and TC formation. Surface cold pools interacting with vertical shear, partly induced by a tilted vortex structure, have been hypothesized to increase the strength and the organization of low-level updrafts (Davis 2015), contributing positively to the area-mean mass flux profile. The relative coldness of the pregenesis boundary layer is a well-observed feature, consistent with at least the presence of cold pools (Zawislak and Zipser 2014). Though cold pools exist in the subcloud layer, they can promote deeper, wider, and more buoyant clouds with higher precipitation rates, which, in turn, leads to stronger cold pools as a positive feedback (Böing et al. 2012). Cold pools allow the scale of convection to increase and the convection to become more organized, occupying a broader area. Cold pool-shear dynamics enhance lowlevel updrafts and their organization, aiding the spinup of the surface vortex eventually (Davis 2015). However, it is unclear if shear-cold pool interactions are essential in the genesis process. The possible effect of cold pools on mass flux profile and updrafts will be investigated. Removal of cold pools through the removal of low-level evaporation cooling is a way to further test the importance of cold pools and the associated mechanism.

As noted in many previous studies, the moisture preconditioning process and the approach to saturation over most of the depth of the troposphere is an important step on the path to TC formation (e.g., Nolan 2007; Wang 2012; Wang et al. 2018). Deep convection is strongly favored in moist patches and suppressed in dry patches (Davis 2015). Dry air, usually introduced through wind shear, is widely accepted to be detrimental to updraft strengthening and tropical cyclogenesis (Smith and Montgomery 2012). Dry air is also suggested to limit moist convection by mainly inhibiting the intensity of updrafts and upward mass flux, but not greatly enhancing the downdrafts and downward mass flux (James and Markowski 2010; Kilroy and Smith 2013). Freismuth et al. (2016) suggested that the entrainment of dry air near $600 \mathrm{hPa}$ in a TC inhibits the updrafts and vertical mass flux and, thus, produces a compromised pouch, allowing more dry-air intrusion. Investigating the effect of dry air can help to address the development of convection and the associated amplifying vorticity in TC genesis as well.

The paper is organized as follows. First, the design of sensitivity experiments is described in section 2; the basic results of the experiments, the characteristics of mass flux, and the evolution of convective updrafts are presented in section 3; section 4 interprets the spinup of the near-surface vortex by diagnosing vorticity and divergence budgets; and summary and conclusions are presented in section 5 .

\section{Methodology}

\section{a. Experimental design}

In the present study, sensitivity experiments are carried out based on the control simulation in Davis (2015), using the same idealized cloud model, the Cloud Model version 1 (CM1) (Bryan and Fritsch 2002; Bryan and Morrison 2012). The model is nonhydrostatic, fully compressible, and integrated on an $f$ plane with doubly periodic lateral boundary conditions. Davis (2015) conducted a 60 -day simulation with uniform sea surface temperature and prescribed uniform background flow. A horizontal 
grid spacing of $3 \mathrm{~km}$ and 66 vertical levels, with the lowest level at $50 \mathrm{~m}$ above the surface, are used in the simulation. The horizontal domain is $960 \mathrm{~km} \times 960 \mathrm{~km}$. The physical parameterization schemes include the Morrison doublemoment microphysical scheme (Bryan and Morrison 2012), the Yonsei University boundary layer scheme (Hong et al. 2006), and the Goddard shortwave and longwave radiation schemes (Chou and Suarez 1999).

To better understand the earliest stage of TC genesis, sensitivity experiments in this study begin 5 days before the end of the control simulation in Davis (2015), when a midtropospheric vortex, the precursor to the TC, can be continuously tracked and the surface TC vortex had not yet formed. The advantage of the current study is that the initial midtropospheric vortex develops in a radiativeconvective equilibrium, and there are no parameters specified a priori that determine its structure. Nevertheless, the results in Davis (2015) are broadly consistent with previous studies that prescribed a vortex, which allows us to explore the sequential processes leading to TC genesis. Note that the point of this paper is not to be an exhaustive list of sensitivity experiments, but rather, a detailed analysis of a small number of permutations designed to alter the lower-tropospheric thermodynamics. The simulation in Davis (2015) is denoted as the control run (CTL) in this study. One sensitivity experiment, denoted EXP_CP, is carried out with identical setup as CTL, except that in EXP_CP, the latent cooling rate associated with precipitation evaporation is reduced by $90 \%$ beneath the cloud base (from the surface to $500-\mathrm{m}$ altitude). To investigate the impact of moisture on convection and, further, on TC formation, continuous vertical wind shear is added to the background wind field, which is expected to introduce dry air. Thus, another sensitivity experiment, EXP_WS, is conducted with the same configuration in CTL, except that the background zonal wind is linearly increased on the whole vertical profile from about $625 \mathrm{~m}$ to $40 \mathrm{~km}$, ensuring the vertical wind shear between 850 and $200 \mathrm{hPa}$ is about $2.5 \mathrm{~m} \mathrm{~s}^{-1}$. We also conducted an experiment with vertical wind shear magnitude of $5.0 \mathrm{~m} \mathrm{~s}^{-1}$ in the same layer. However, a TC did not form even after $170 \mathrm{~h}$ of integration, nor did the maximum 10-m wind speed (less than $17 \mathrm{~m} \mathrm{~s}^{-1}$ ) exhibit an increasing trend. Thus, the magnitude of $2.5 \mathrm{~m} \mathrm{~s}^{-1}$ is chosen in this paper to investigate the impact of dry air on TC formation without totally preventing TC formation. All the simulations integrate for $144 \mathrm{~h}$, beyond the original 120 -h time limit, to give a tropical cyclone more time to develop. To make sure that the differences between these experiments are statistically significant, an ensemble of five simulations for each experiment is also conducted by adding potential temperature perturbations of up to $0.2 \mathrm{~K}$, which is random at every grid point, from the surface to a height of $500 \mathrm{~m}$. The inclusion of potential temperature perturbations to the lower troposphere leads to modest differences in TC genesis and helps to identify the differences caused by changed thermodynamic fields in EXP_CP and EXP_WS that are statistically meaningful. The 144-h integrating period is denoted as $t=0-144 \mathrm{~h}$ in the following analyses.

\section{b. Vorticity budget equation}

Given the importance of vorticity amplification process during TC genesis, a vorticity budget equation is employed to better understand the process of vortex spinup. The vorticity budget equation in the expression of temporal change of the circulation $C$ in Davis and Galarneau (2009) is adopted in this paper:

$$
\begin{aligned}
\frac{\partial C}{\partial t}= & \underbrace{-\oint \eta \mathbf{V} \cdot \hat{\mathbf{n}} d l}_{\text {Convergence }}+\underbrace{\oint \omega\left(\hat{\mathbf{k}} \times \frac{\partial \mathbf{V}}{\partial p}\right) \cdot \hat{\mathbf{n}} d l}_{\text {Tilting }} \\
& +\underbrace{\oint(\hat{\mathbf{k}} \times \mathbf{F}) \cdot \hat{\mathbf{n}} d l}_{\text {Friction }},
\end{aligned}
$$

where $\eta$ is the absolute vertical vorticity, $\mathbf{V}$ is the stormrelative horizontal wind vector, $\omega$ is the vertical velocity on isobaric coordinates, $\hat{\mathbf{k}}$ is the unit vector in the vertical direction, and $\mathbf{F}$ is the friction vector. Equation (1) contains closed line integrals evaluated around a square box; thus, $\hat{\mathbf{n}}$ is the unit vector outward normal to the edges of the box, and $d l$ is the differential along the perimeter of the box. The contribution to the local change in circulation is separated into three terms on the right-hand side of Eq. (1): the convergence of the horizontal advective flux of vertical vorticity, the tilting term, and the friction term, respectively. The friction term includes the effect of the planetary boundary layer parameterization and is directly output from the model.

\section{c. Divergence budget equation}

The amplification of vorticity and the process of vortex spinup are usually accompanied by low-level convergence. A divergence budget is employed here to investigate the key processes in the low-level convergence mechanism. The divergence budget is as follows:

$$
\begin{aligned}
\frac{\partial \delta}{\partial t}= & \underbrace{\mathbf{V} \cdot \nabla \delta}_{\mathrm{HAD}}-\underbrace{\omega \frac{\partial \delta}{\partial p}}_{\mathrm{VAD}}-\underbrace{\nabla^{2} \Phi}_{\mathrm{PG}}-\underbrace{\nabla \omega \frac{\partial \mathbf{V}}{\partial p}}_{\mathrm{EVS}}-\underbrace{\delta^{2}}_{\mathrm{SD}} \\
& +\underbrace{2 J(u, v)}_{\mathrm{DHF}}+\underbrace{f \zeta-(\hat{\mathbf{k}} \times \mathbf{V}) \cdot \nabla f}_{\mathrm{RE}}+\underbrace{\nabla \cdot \mathbf{F}}_{\mathrm{F}},
\end{aligned}
$$

where $\delta$ is the horizontal divergence, $\zeta$ is the relative vertical vorticity, $\Phi$ is the geopotential height, $J(u, v)$ is 

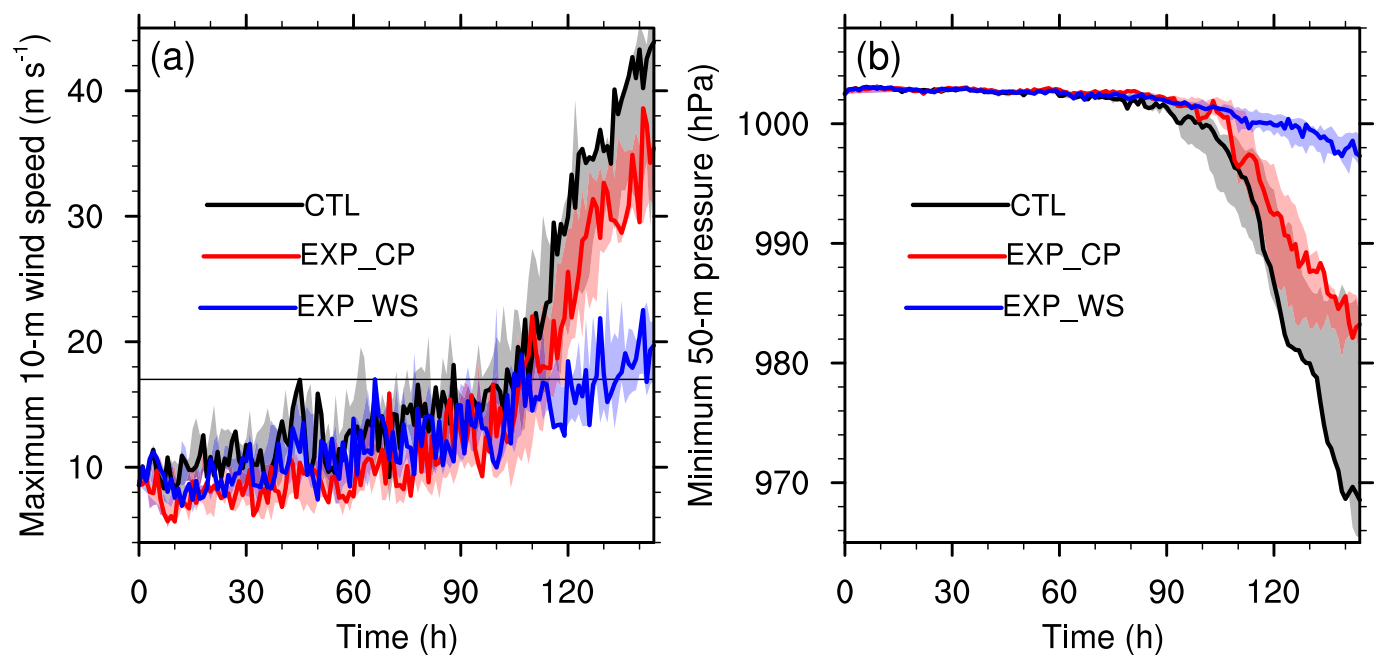

FIG. 1. Time series of (a) maximum 10-m wind speed ( $\mathrm{m} \mathrm{s}^{-1}$ ) and (b) minimum 50-m pressure (hPa). The gray-, red-, and blue-shaded areas represent the ranges of ensemble simulation results of CTL (thick black line), EXP_CP (thick red line), and EXP_WS (thick blue line), respectively. The horizontal gray line in (a) denotes $17 \mathrm{~m} \mathrm{~s}^{-1}$, which is the minimum wind speed of a tropical storm.

the Jacobian $(\partial u / \partial x)(\partial v / \partial y)-(\partial v / \partial x)(\partial u / \partial y)$ of horizontal wind $(u, v)$, and $f$ is the Coriolis parameter. The term on the left-hand side of Eq. (2) is the tendency of divergence, while the first six terms on the right-hand side (RHS) are the horizontal (HAD) and vertical advection (VAD) of divergence, the change of the gradient of isobaric layer or the gradient of pressure gradient (PG), the effect of vertical shear of horizontal wind (EVS), the square of divergence (SD), and the deformation of horizontal flow (DHF). The last two terms are the rotation effect (RE) and the friction term (F). The friction also includes the boundary layer parameterization effect and is directly output from the model.

\section{Results of control and sensitivity experiments}

\section{a. TC genesis and vorticity evolution}

Figure 1a shows the time series of the maximum 10-m wind speed in all experiments. The maximum wind speed is actually the maximum instantaneous wind anywhere in the $960 \mathrm{~km} \times 960 \mathrm{~km}$ domain, which can be used to identify the evolution of TC intensity. The wind speed grows in sensitivity experiments with reduced cold pools (EXP_CP) and extra vertical wind shear (EXP_WS), though more slowly than CTL, eventually reaching $17 \mathrm{~m} \mathrm{~s}^{-1}$, the threshold of tropical storm (TS) strength and our definition of genesis completion. Note that the wind speed in EXP_CP exhibits a significant intensification rate analogous to that in CTL once reaching TS, while that in EXP_WS still grows quite slowly though its wind speed generally exceeds $17 \mathrm{~m} \mathrm{~s}^{-1}$ by $120 \mathrm{~h}$. The evolution of the minimum pressure at $50-\mathrm{m}$ altitude
(Fig. 1b) also shows distinct differences among the three experiments: TC intensification in EXP_CP is postponed for a few hours, while the developing rate in EXP_WS is significantly suppressed.

The ranges of the maximum 10-m wind speed and the minimum 50-m pressure in ensemble simulations are presented as shaded areas in Figs. 1a and 1b, respectively. The inclusion of potential temperature perturbations in the initial condition of ensemble simulations leads to small differences in the intensification beginning time, the intensification rate, and the TC strength compared with the original experiments, CTL, EXP_CP, and EXP_ WS, respectively. Such differences may be attributed to the stochastic nature of convection in the model. A similar development delay or suppression relative to CTL can still be clearly recognized in ensemble experiments, which represents the features of different sensitivity experiments very well.

Figure 2 gives the horizontal cross sections of surface virtual temperature, surface horizontal wind field, and $2-\mathrm{km}$ vertical velocity after TC formation in the three experiments. It can be seen that among the three experiments, when the TC forms, the surface atmosphere in CTL is the coldest and that in EXP_CP is the warmest because of the reduced cold pool effect. Impacted by the vertical wind shear, the TC in CTL_WS forms farther east. Moreover, the circulation is much more asymmetric, with intense convective cells and the near-surface cold core located in the downshear quadrant (east) of the TC center (Fig. 2c), while the coldest areas in the other experiments are located basically near the TC center (Figs. 2a,b). 

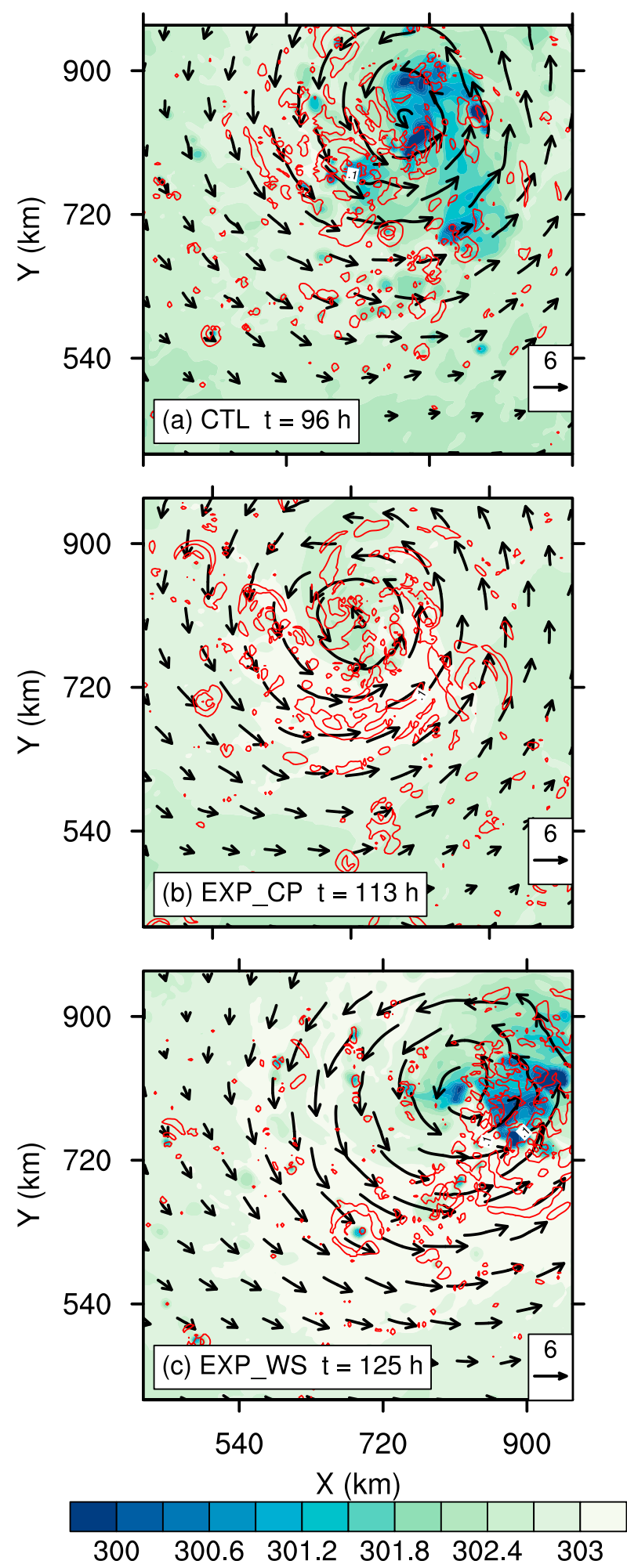

FIG. 2. Horizontal cross sections of 50-m virtual temperature (shaded; K), 50-m horizontal wind field (vectors; $\mathrm{m} \mathrm{s}^{-1}$ ), and 2-km vertical velocity (red contours; $>0.1 \mathrm{~m} \mathrm{~s}^{-1}$ ) in (a) CTL at $t=96 \mathrm{~h}$, (b) EXP_CP at $t=113 \mathrm{~h}$, and (c) EXP_WS at $t=125 \mathrm{~h}$.
During the simulation, a midtropospheric vortex can be tracked by the maximum vorticity at $6 \mathrm{~km}$ averaged within a box of $150 \mathrm{~km} \times 150 \mathrm{~km}$. The time-height depiction of relative vorticity, which is averaged within the subdomain that defines the midtropospheric vortex $(150 \mathrm{~km} \times 150 \mathrm{~km})$, indicates that the strong positive vorticity center gradually moves downward from the middle troposphere $(z=4-8 \mathrm{~km})$ to the near surface obviously in CTL and EXP_CP (Figs. 3a,b). The areamean vorticity center within the subdomain following the low-level vortex also shows the similar downward shift (Figs. 3d-f). The low-level vortex center is identified by the maximum $1-\mathrm{km}$ vorticity averaged in a $72 \mathrm{~km} \times 72 \mathrm{~km}$ box area, representing the low-level vortex area. The low-level vortex becomes obvious after $t=60 \mathrm{~h}$. Note that in EXP_WS (Figs. 3c,f), the evolution of area-mean lower-tropospheric vorticity is quite different when the subdomain moves with the midtropospheric vortex or the lower-tropospheric vortex, owing to the spatial displacement of the vortex at these two levels induced by vertical wind shear.

Figure 4 shows the time series of vorticity at different altitudes in three experiments and their ensemble results. It can be seen that the increase of 1-km vorticity begins at $t=40 \mathrm{~h}$, followed by remarkable increases of the near-surface and the $1-\mathrm{km}$ positive vorticity beginning at about $t=80 \mathrm{~h}$ in CTL, following the steady vorticity amplification at the altitude of $6 \mathrm{~km}$ (Fig. 4a). In EXP_CP, the intensity of vorticity is weaker, and the amplification timing of the lower-tropospheric vorticity is later compared with CTL (Figs. 3b,e). A significant increase of $1-\mathrm{km}$ vorticity begins at about $t=82 \mathrm{~h}$ (Figs. 4c,d), also following the increase of $6-\mathrm{km}$ vorticity. In EXP_WS, the positive vorticity above the lower troposphere is much weaker (Figs. 3c,f). The lowlevel cyclonic vorticity increases early (Fig. 4f), and the vortex struggles to intensify thereafter. However, the evolution of midlevel vorticity shows that the midtropospheric vortex fails to intensify (Figs. 4e,f). Below, we will investigate how the spatial displacement of the midtropospheric and the lower-tropospheric vortex centers leads to this reduced intensification rate.

Since a moist environment through a deep tropospheric layer is necessary for TC genesis, the relative humidity $(\mathrm{RH})$ in all experiments is diagnosed. The cross sections of area-averaged $\mathrm{RH}$ show that the humidifying at lower troposphere (about $1-4 \mathrm{~km}$ ) is suppressed in sensitivity experiments, especially in EXP_WS (Fig. 5). In CTL, lower-tropospheric RH exceeds $80 \%$ after $t=40 \mathrm{~h}$. However, the RH does not reach $80 \%$ until about $t=82 \mathrm{~h}$ in EXP_CP and until $t=110 \mathrm{~h}$ in EXP_WS (Figs. 4c,e). The timing of $1.5-\mathrm{km} \mathrm{RH}$ exceeding $80 \%$ coincides well with the increase of low-level vorticity 

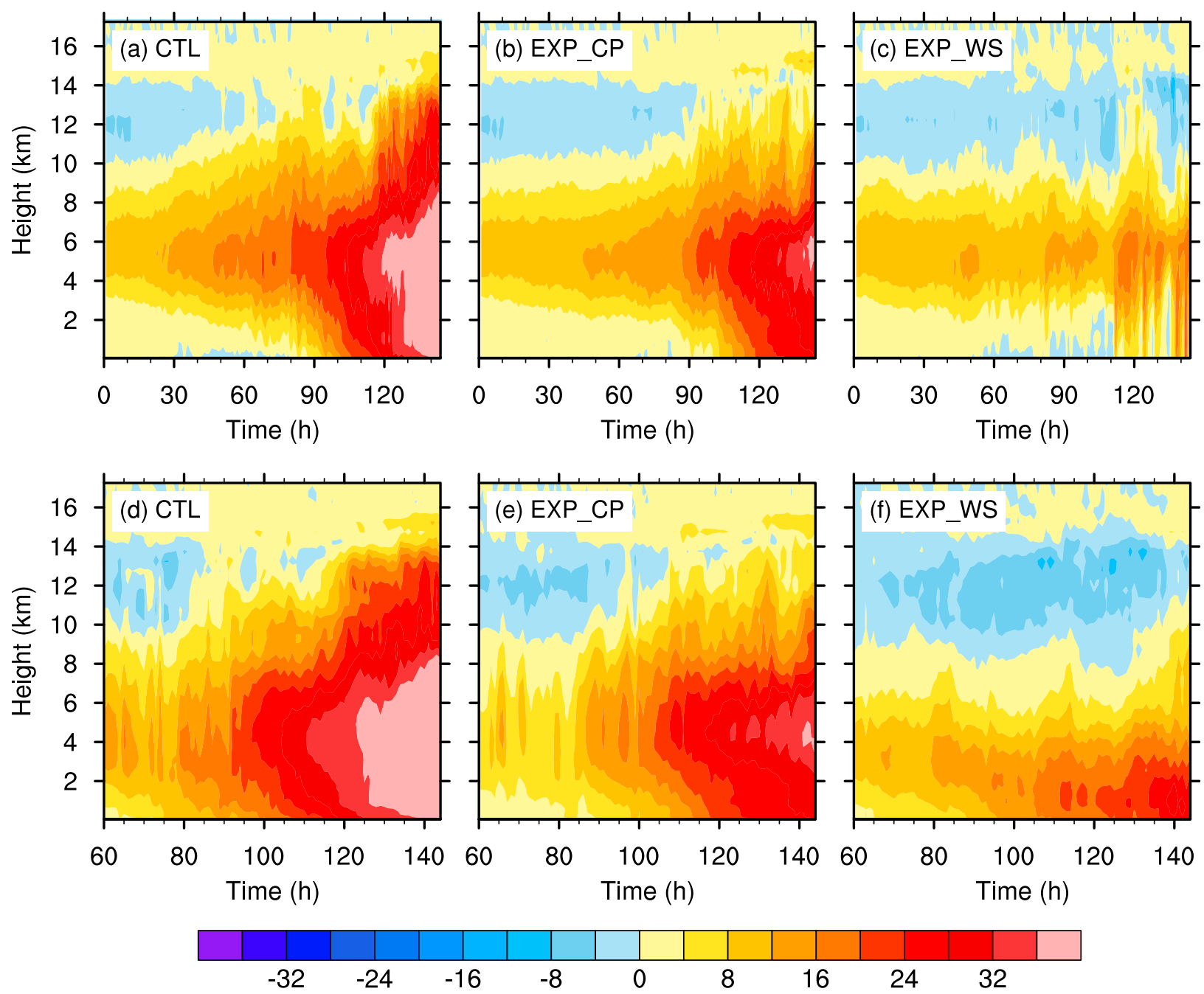

FIG. 3. Vortex-following time-height cross sections of area-mean relative vorticity $\left(10^{-5} \mathrm{~s}^{-1}\right)$ in (a),(d) CTL, (b),(e) EXP_CP, and (c),(f) EXP_WS. The area is a $150 \mathrm{~km} \times 150 \mathrm{~km}$ box centered on the vortex at (a)-(c) 6 and (d)-(f) $1 \mathrm{~km}$. Note that the time window plotted for (d)-(f) begins at $60 \mathrm{~h}$.

(e.g., Figs. 4c,e) and the near-saturated column through the lower to middle troposphere in Fig. 4 corresponds with significant amplification of relative vorticity. In CTL_WS, however, the 6-km RH is rather low following the lower-tropospheric vortex because of the dry air being advected above the low-level vortex.

The relatively dry air in CTL_WS is likely because of the wind shear. A swarm of 20 -h backward trajectories of parcels starting at $1-2-\mathrm{km}$ altitude at $t=60 \mathrm{~h}$ is given in Fig. 6. The parcels in CTL generally swirl cyclonically into the target box during $t=40-60 \mathrm{~h}$ (Fig. 6a). The parcels in EXP_WS generally move eastward into the box under the influence of eastward wind shear (Fig. 6b), corresponding well with the relative eastward location of the TC vortex (Fig. 2c). Basically, the parcels in CTL contain more water vapor (Figs. 6a,b). Figure $6 c$ gives the histogram of the accumulated number of parcels with different water vapor contents on the trajectories, suggesting that there are more parcels containing higher moisture in CTL. The impact of slower humidification of low-level atmosphere on the spinup of a near-surface vortex will be discussed in the following sections.

\section{b. Structure of the mass flux}

Following the midlevel vortex, a notable increase in the 6-km vertical mass flux occurs at $t=38 \mathrm{~h}$ (Fig. 7a), while the vertical mass flux at $2 \mathrm{~km}$ does not become generally positive until roughly $t=72 \mathrm{~h}$ in CTL (Figs. 7a, b). In EXP_CP, a notable increase in the 6-km mass flux and positive transition in the $2-\mathrm{km}$ mass flux occur at about $t=60$ (Fig. 7c) and $t=82 \mathrm{~h}$ (Figs. 7c,d), respectively. In EXP_WS, a distinct increase of 6-km mass 

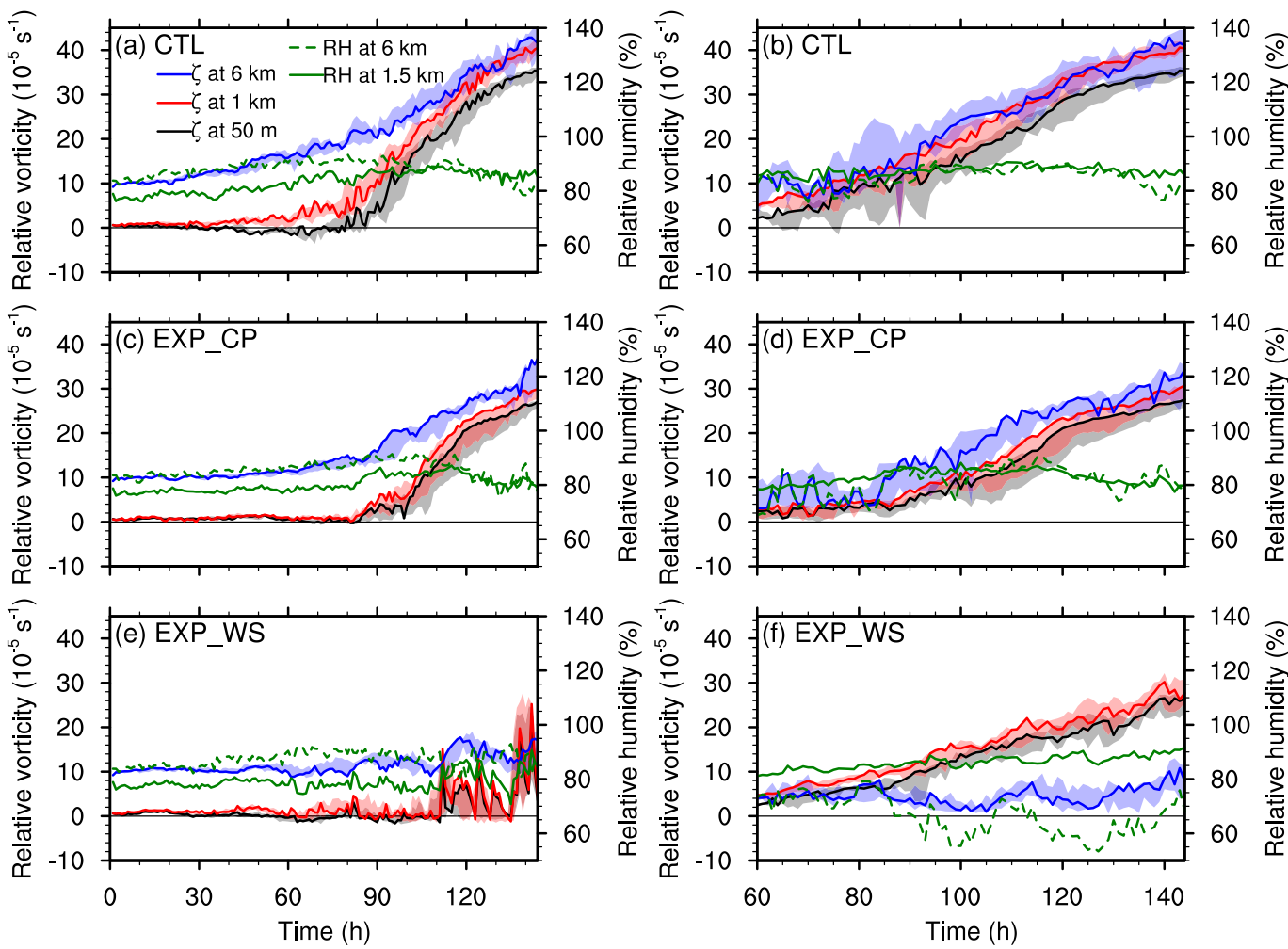

FIG. 4. Time series of area-mean relative vorticity $\left(10^{-5} \mathrm{~s}^{-1}\right)$ at $6-\mathrm{km}$ (blue line), 1-km (red line), and 50-m (black line) altitudes and relative humidity at 1.5- (green solid line; \%) and 6-km altitudes (green dashed line) in a $150 \mathrm{~km} \times$ $150 \mathrm{~km}$ box centered on the vortex at (a),(c),(e) 6 and (b),(d),(f) $1 \mathrm{~km}$ in (a),(b) CTL, (c),(d) EXP_CP, and (e),(f) EXP_WS. The gray-, red-, and blue-shaded areas represent the ranges of ensemble simulations.

flux occurs at about $t=72 \mathrm{~h}$ (Fig. 7e), after when the 2-km mass flux also becomes generally positive (Fig. 7f). However, the mass fluxes at lower and middle troposphere undergo a steady increase thereafter, weaker than those in CTL and EXP_CP. The results of the ensemble simulations also present the sequential increase of mass flux from midtroposphere to lower troposphere. The ensemble of the mass flux profiles shows that the 2-km mass flux in CTL and EXP_CP intensifies significantly during $t=81-120 \mathrm{~h}$ following the lower-tropospheric vortex (Figs. 8b,d), which is much larger than that in EXP_WS (Fig. 8f). Moreover, the low-level upward mass flux in EXP_CP and EXP_WS increases soon after the deep-layer humidity within the vortex exceeds $80 \%$ (Figs. 5, 7).

Previous studies (e.g., Montgomery et al. 2006) shows that low-level vorticity amplification is usually dominated by the stretching effect of convection. Gjorgjievska and Raymond (2014) also suggested that a bottom-heavy mass flux profile that exhibits a strong positive vertical gradient in the shallow layer above the surface can induce vorticity convergence in the boundary layer. The timing of the low-level mass flux increase is consistent with the positive vorticity amplification at $1 \mathrm{~km}$ (Figs. 4, 7), suggesting that vortex spinup near and slightly above the top of the boundary layer is attributed to the mass flux and the corresponding strong convergence (Raymond and López-Carrillo 2011). We will show this explicitly using a vorticity budget in section 4 .

Figure 8 shows that the shape of mass flux profiles is not technically bottom-heavy, which is consistent with the result in Kilroy et al. (2018) that the mass flux profile does not need to be bottom-heavy for genesis and intensification to occur. However, the positive vertical gradient of low-level mass flux in CTL is the largest among three experiments during $t=41-80 \mathrm{~h}$ (Fig. 8a). Because of the reduced evaporative cooling rate, cool downdrafts near the surface and their corresponding surface cold pools are suppressed in EXP_CP. Thus, fewer updrafts are triggered by weaker cold pools. As a result, the low-level upward mass flux and the associated vertical gradient in EXP_CP before $t=81 \mathrm{~h}$ is the weakest (Fig. 8c). After this time, the low-level vertical mass flux in EXP_CP eventually increases to the intensity of CTL (Figs. 8b,d). The comparison between CTL and EXP_CP indicates that the effect of cold pools 

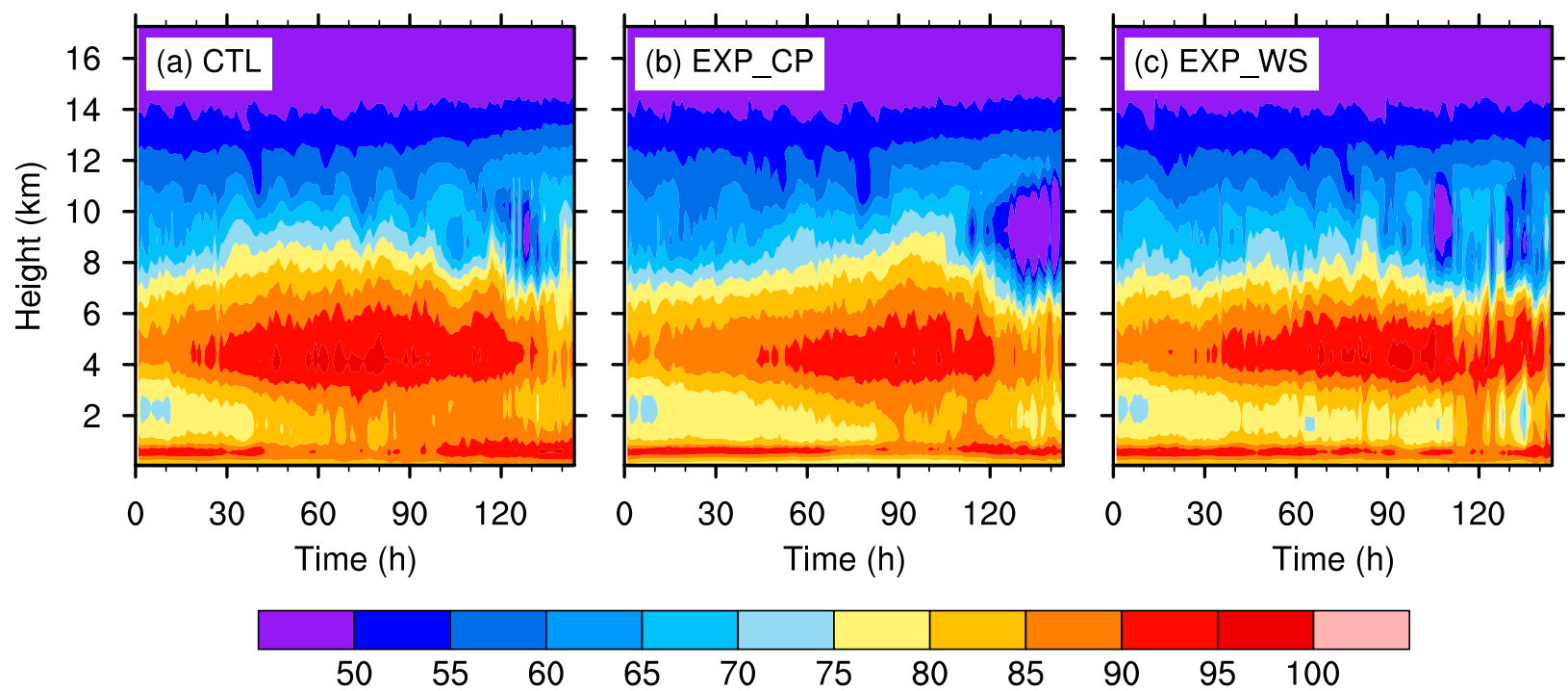

FIG. 5. Vortex-following time-height cross sections of area-mean relative humidity (\%) in (a) CTL, (b) EXP_CP, and (c) EXP_WS. The area is a $150 \mathrm{~km} \times 150 \mathrm{~km}$ box centered on the vortex at $6 \mathrm{~km}$.

can enhance the low-level vertical gradient of mass flux and cause strong convergence above the boundary layer earlier. Because of the shear and horizontal displacement of lower-level and midlevel vortices, drier environment air infiltrates the vortex. As a result, the upward mass flux is much weaker and amplifies more slowly in EXP_WS. Thus, although cold pools exist in EXP_WS, the vertical gradient of low-level mass flux in EXP_WS is still the weakest (Fig. 8e). Raymond and Sessions (2007) suggested that a moistening of an air column only changes the magnitude of mass flux but not the mass flux profile shape. However, different from those in CTL, the mass flux profiles in EXP_WS show weak upward mass flux above the lower troposphere, and the vertical gradient of mass flux does not show significant increase, which has implications for the intensification of a shallow vortex.

The surface heat flux in sensitivity experiments is also suppressed in terms of latent heat flux and sensible heat flux, which is partly responsible for the reduced mass flux. According to Gjorgjievska and Raymond (2014), the larger surface entropy flux helps foster the larger mass flux magnitude. In EXP_CP, reduced evaporative cooling rate suppresses surface cold pools (Fig. 2b). As a result, the surface sensible heat flux in EXP_CP is reduced (Fig. 9a) because of smaller temperature difference between the sea surface and the near-surface atmosphere. However, the surface latent heat flux in EXP_CP exhibits similar rate of increase as that in CTL after $t=80 \mathrm{~h}$ (Fig. 9b), which is associated with the significant intensification of the surface wind (Fig. 1a). The differences of the surface sensible and latent heat fluxes between CTL and EXP_WS gradually increase after $t=45 \mathrm{~h}$ (Fig. 9) under the influence of slower wind speed in EXP_WS. Similarly, the notable increases of surface heat flux in three experiments also occur around the moment of the low-level humidity exceeding $80 \%$.

\section{c. Characteristics of convection}

Davis (2015) suggested that it is the area coverage of updrafts that mainly dominates the increase of the lowlevel upward mass flux before genesis. The number of updrafts, downdrafts, and the ratio of updraft to downdraft at $2-\mathrm{km}$ altitude in the three experiments is presented in Fig. 10. The updrafts and downdrafts here are defined to be greater than $0.5 \mathrm{~m} \mathrm{~s}^{-1}$ in magnitude. The number of both updrafts and downdrafts is fewer than 20 in the beginning of the simulation, and then they both increase with TC genesis (Figs. 10a,b) as a result of convection development. In all the experiments, the updraft-downdraft ratio is greater than 1.0, indicating that updrafts are already more widespread than downdrafts. However, the 2-km mass flux does not become generally positive until significant increases of both updrafts and downdrafts numbers occur at $t=72 \mathrm{~h}$ in CTL and $t=82 \mathrm{~h}$ in EXP_CP (Figs. 10a,b). The development of updrafts and downdrafts in EXP_WS, whose increases occur as early as those in CTL, are much slower than those in the other two experiments. Note that the numbers of updrafts and downdrafts still decrease intermittently after $t=110 \mathrm{~h}$ even though their ratio remains greater than 1.0 in EXP_WS, indicating that the development of convection is interrupted by dry air at 

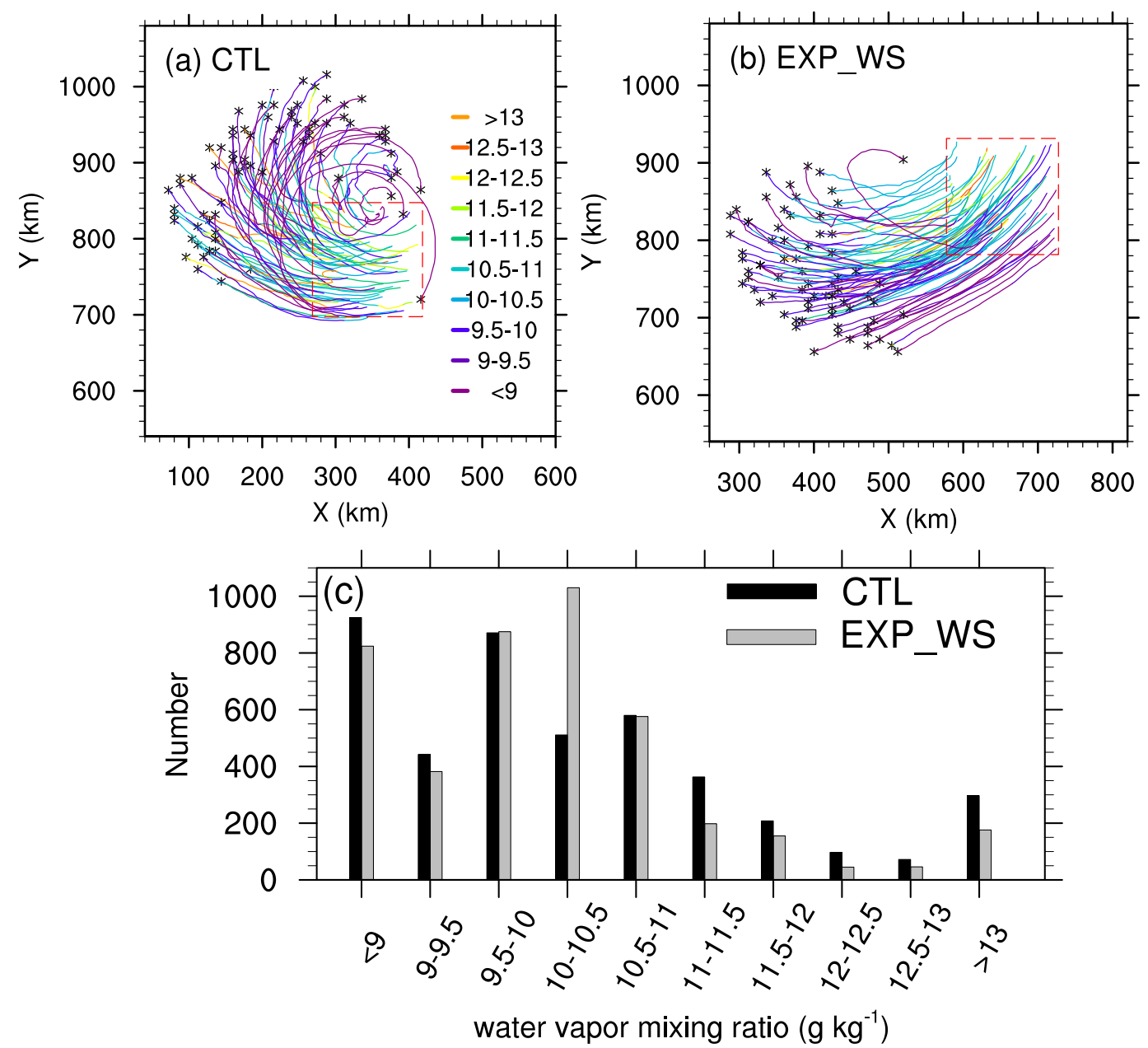

FIG. 6. The 20-h backward trajectories of parcels starting at $1-2-\mathrm{km}$ altitude at $t=60 \mathrm{~h}$ in a $150 \mathrm{~km} \times 150 \mathrm{~km}$ box (dashed red box) centered on the vortex at 6-km altitude in (a) CTL and (b) EXP_WS. The colors of trajectories denote water vapor mixing ratio $\left(\mathrm{g} \mathrm{kg}^{-1}\right)$, and the black stars denote the positions of the parcels at $t=40 \mathrm{~h}$. (c) The accumulated number of parcels in the trajectories of (a) and (b) with different water vapor mixing ratios.

the low troposphere and the vertical incoherence of the vortices.

The above results indicate that the dominant role of updrafts in terms of coverage alone does not determine the upward mass flux increasing. The enhancement of convection intensity in terms of increasing number of strong updrafts and downdrafts might be another factor.

Further, the characteristics of convective cores are diagnosed. Each convective core is selected as follows: the maximum vertical velocity at $z=1-5 \mathrm{~km}$ on a grid point is positive, and the vertical velocity on the point is the maximum within the $30 \mathrm{~km} \times 30 \mathrm{~km}$ box around it. Thus, the grid point is identified as the convective core. The time series of the vertical velocity on the strongest convective core at every hour indicates that the convection in the three experiments gradually strengthens (Fig. 10d). The intensity of convective cells in EXP_CP remains weak at the beginning and then increases after about $t=73 \mathrm{~h}$. Though the initial convective cells in EXP_WS are even stronger than CTL, they intensify rather slowly during the whole TC genesis process. In summary, convective cells gradually grow into ones with stronger intensity in environments approaching near saturation, and those strong updrafts generally occupy dominant coverage relative to downdrafts, together promoting low-level net upward mass flux and vorticity amplification.

Since similar evolutions of convection intensity also occur in EXP_CP, in which the TC vortex spins up nearly as quickly as that in CTL, while the convection is suppressed and TC formation is delayed in EXP_WS, one can infer that the low-level humidity rather than the strong cold pools is essential to convection enhancement and TC formation. Since Davis (2015) suggested that the 


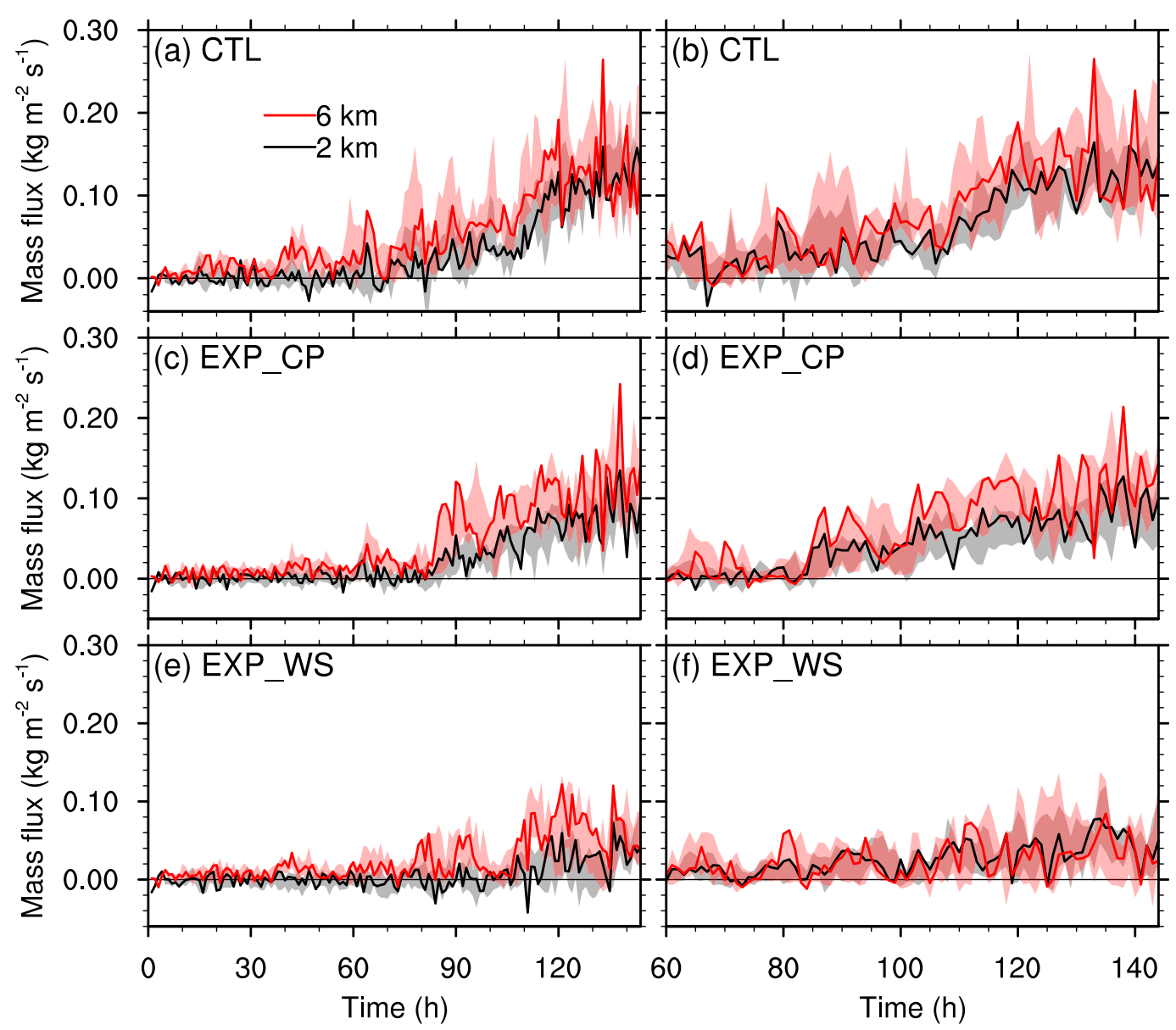

FIG. 7. Time series of area-mean vertical mass flux $\left(\mathrm{kg} \mathrm{m}^{-2} \mathrm{~s}^{-1}\right)$ at 6 - (red line) and 2-km (black line) altitudes in a $150 \mathrm{~km} \times 150 \mathrm{~km}$ box centered on the vortex at (a),(c),(e) 6 and (b),(d),(f) $1 \mathrm{~km}$ in (a),(b) CTL, (c),(d) EXP_CP, and (e),(f) EXP_WS. The gray- and red-shaded areas represent the ranges of ensemble simulations. The horizontal gray line denotes $0 \mathrm{~kg} \mathrm{~m}^{-2} \mathrm{~s}^{-1}$.

cold pool-shear dynamics could enhance the low-level updrafts and their organization, we further diagnose the area-mean difference between $50-\mathrm{m}$ and $2-\mathrm{km}$ virtual potential temperature (Fig. 11), which represents lowlevel buoyancy to a certain extent. It can be seen that the virtual potential temperature difference in EXP_CP is the smallest, indicating that a near-surface parcel can be more easily lifted and develop into deep convection. Thus, we suspect that the reduced ability of cold pools to trigger and organize updrafts is compensated by a reduction in stratification of the environment. While emphasizing the impact of relative humidity on convection, we should not exclude the humidifying effect of convection. The comparison of CTL and EXP_WS (Fig. 11) suggests that the quickly humidifying environment in CTL somewhat offsets the lower buoyancy effect generally, resulting in earlier and stronger convection enhancement in turn. In EXP_WS, because of the horizontal displacement of midtropospheric and lower-troposphere vortices, trajectories with drier air can enter the vortex, thereby reducing convective intensity and coverage.

\section{Analysis of low-level vorticity amplification}

\section{a. Evolution of vorticity at early stage}

In this section, we focus on the spinup of the vortex slightly above (2-km altitude) and in (50-m altitude) the boundary layer during TC genesis. Smith et al. (2009) suggested that the maximum tangential wind speed is located near the top of the boundary layer. The actual top of the boundary layer is close to $1 \mathrm{~km}$; hence, we examine the 2-km level to be safely above the top of the boundary layer. Our results are not sensitive to this choice. Figure 12 shows the time series of the relative vorticity and the divergence at $2-\mathrm{km}$ and $50-\mathrm{m}$ altitude, which are averaged in a box area using the 6-min model output. The box length of $102 \mathrm{~km}$ is chosen in order to cover the major part of the mesoscale low pressure 

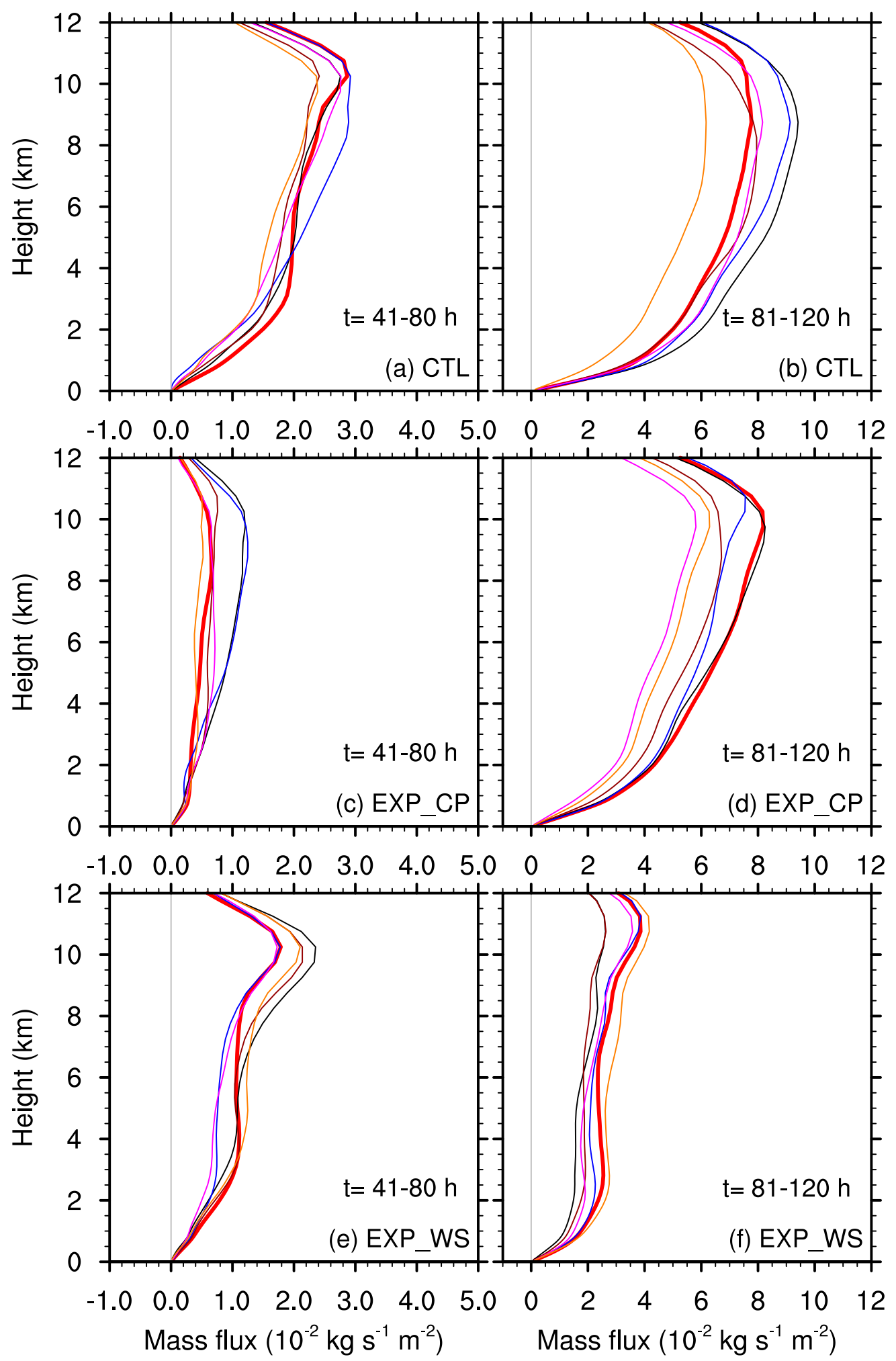

FIG. 8. Vertical profiles of temporal-mean [(a),(c),(e) $t=41-80$ and (b),(d),(f) $t=81-120 \mathrm{~h}$ ) and area-mean vertical mass flux $\left(10^{-2} \mathrm{~kg} \mathrm{~s}^{-1} \mathrm{~m}^{-2}\right)$ in a $150 \mathrm{~km} \times 150 \mathrm{~km}$ box centered on the vortex at $1 \mathrm{~km}$ in (a),(b) CTL (red line), (c),(d) EXP_CP (red line), (e),(f) EXP_WS (red line), and their ensemble simulations (black lines). The vertical gray line denotes $0 \mathrm{~kg} \mathrm{~s}^{-1} \mathrm{~m}^{-2}$. 

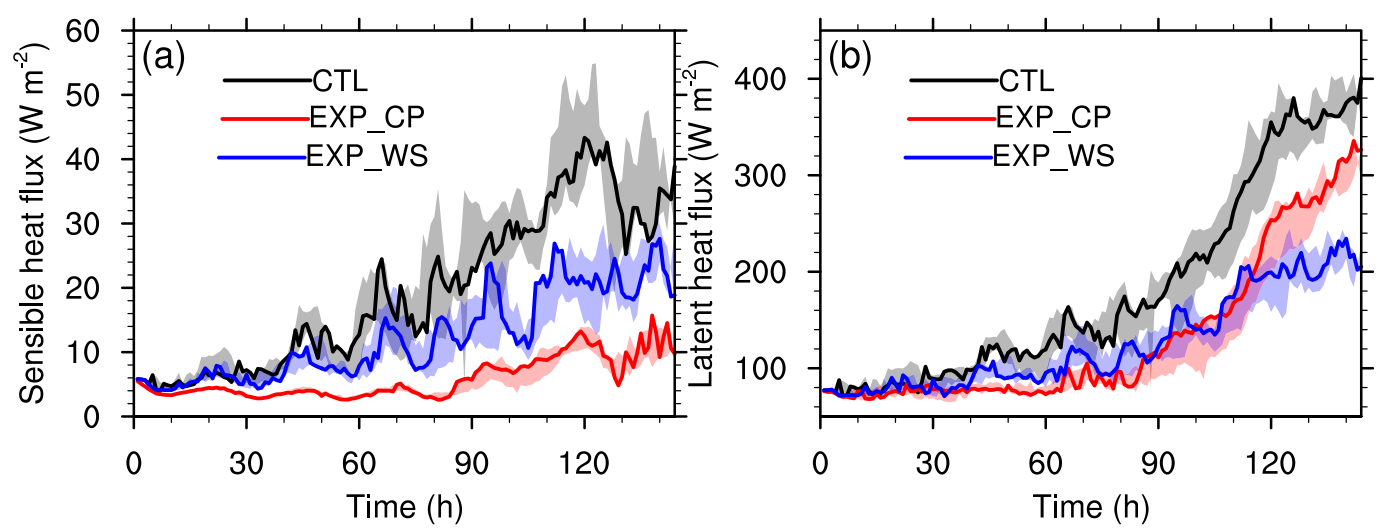

FIG. 9. Time series of area-mean (a) surface sensible heat flux $\left(\mathrm{W} \mathrm{m}^{-2}\right)$ and (b) surface latent heat flux $\left(\mathrm{W} \mathrm{m}^{-2}\right)$ in a $150 \mathrm{~km} \times 150 \mathrm{~km}$ box centered on the vortex at $1 \mathrm{~km}$ in CTL (black line), EXP_CP (red line), and EXP_WS (blue line). The gray-, red-, and blue-shaded areas represent the ranges of ensemble simulations.

system during the whole simulation. The box here is moving with the maximum $1-\mathrm{km}$ vorticity averaged in a $72 \mathrm{~km} \times 72 \mathrm{~km}$ box area, representing the lowertropospheric vortex area. The vortex center at $1-\mathrm{km}$ altitude approximately represents those at $2-\mathrm{km}$ and $50-\mathrm{m}$ altitude, and we choose $72 \mathrm{~km}$ as the box length because of the small size of the initial vortex. We also compute a representative pressure gradient as the difference between area-averaged pressure in the ring of radius of $34-51 \mathrm{~km}$ and in the circular region with radius of $17 \mathrm{~km}$. It shows that the $2-\mathrm{km}$ vorticity does increase prior to the amplification of 50-m vorticity (Figs. 12a-c).
However, the pressure gradient and the corresponding convergence near the surface $(50 \mathrm{~m})$ eventually exceed those above the boundary layer $(2 \mathrm{~km})$, indicating stronger radial inflow near the surface. Among the three experiments, the increase of low-level vorticity in CTL is the earliest and the most significant (Figs. 12a-c). The 2-km vorticity in EXP_WS also increases very early. However, while the vorticity in CTL and EXP_CP exhibits remarkable amplification later as the pressure gradient rapidly increases, the vorticity amplifies rather steadily and slowly in EXP_WS, consistent with the slow vortex spinup near the surface.
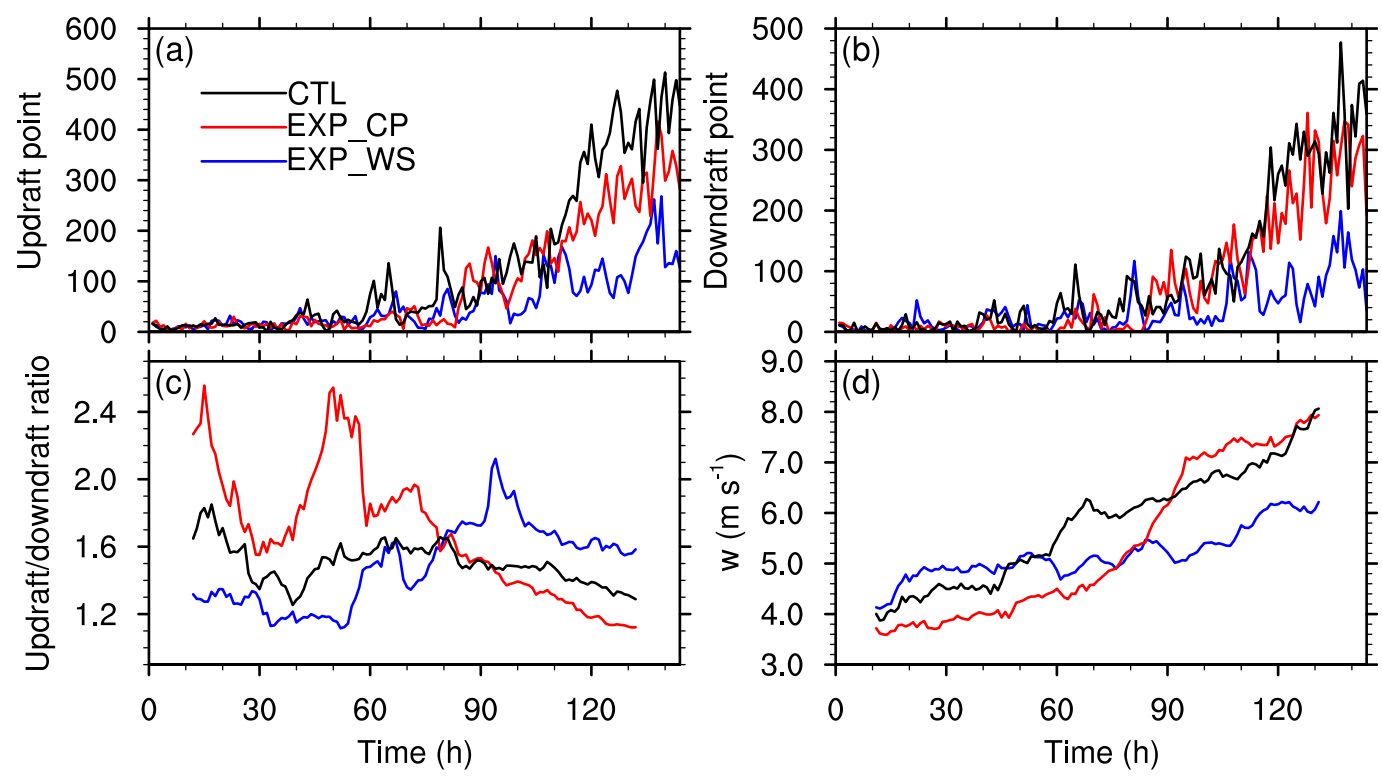

FIG. 10. Time series of (a) number of updrafts and (b) number of downdrafts (grid points where the vertical wind speed exceeds a magnitude of $0.5 \mathrm{~m} \mathrm{~s}^{-1}$ ) at $2 \mathrm{~km}$, (c) the 24-h running-averaged ratio of 2-km updrafts to downdrafts (each exceeding $0.5 \mathrm{~m} \mathrm{~s}^{-1}$ ), and (d) the 24-h running-averaged $2-\mathrm{km}$ vertical velocity $\left(\mathrm{m} \mathrm{s}^{-1}\right)$ on the core of the strongest cell in a $150 \mathrm{~km} \times 150 \mathrm{~km}$ box centered on the vortex at $1 \mathrm{~km}$ in CTL (black), EXP_CP (red), and EXP_WS (blue). 


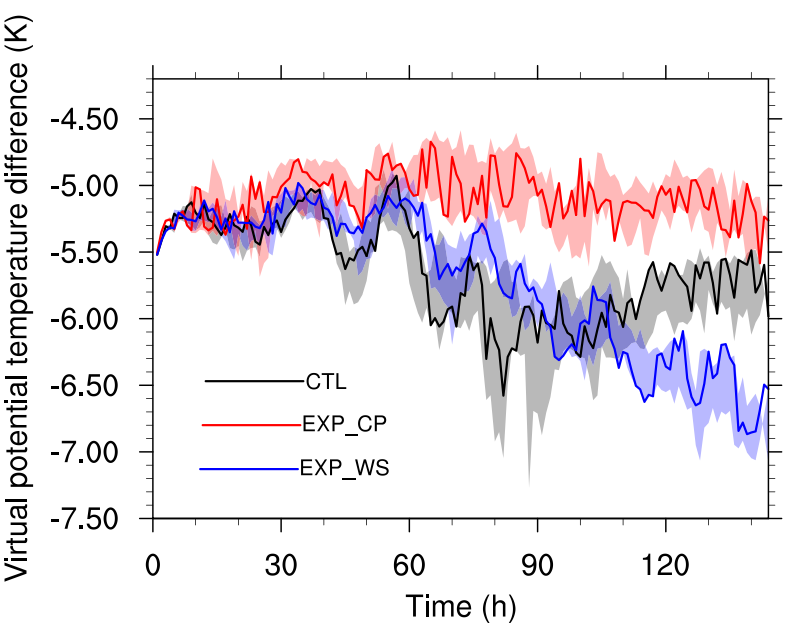

FIG. 11. Time series of the area-mean difference between 50-m and $2-\mathrm{km}$ virtual potential temperature $(\mathrm{K})$ in a $150 \mathrm{~km} \times 150 \mathrm{~km}$ box centered on the vortex at $1 \mathrm{~km}$ in CTL (black line), EXP_CP (red line), and EXP_WS (blue line). The gray-, red-, and blueshaded areas represent the ranges of ensemble simulations.

Figures $12 \mathrm{~d}-\mathrm{f}$ give more details about the sequential evolution of vorticity and the pressure gradient in the very early stage of TC genesis. In CTL, the $2-\mathrm{km}$ vorticity slightly increases, induced by weak convergence above the boundary layer, consistent with the generally positive pressure gradient at $2 \mathrm{~km}$ (Fig. 12d). According to section 3 , those low-level convective cells gradually become stronger, occupied by more updrafts instead of more downdrafts in the near-saturated environment. The associated increasing upward mass flux and vertical gradient of mass flux produce convergence at low levels and favor the growth of $2-\mathrm{km}$ positive vorticity, in which the frictional effect is not important. The spinup above the boundary may be interpreted by balanced dynamics (Smith et al. 2009).

After the initial vorticity amplification and the pressure gradient become generally positive above the boundary layer, the pressure gradient within the boundary layer also becomes positive, and distinct convergence occurs after $t=\sim 53 \mathrm{~h}$ in CTL (Fig. 12d). Though the $50-\mathrm{m}$ pressure gradient becomes negative occasionally, it still induces significant convergence near the surface on average, which makes 50-m vorticity increase rapidly. This result is consistent with the statement by Smith et al. (2009) in which the spinup of the near surface vortex follows the spinup of the tangential wind/vortex above the boundary layer. In EXP_CP, the 2-km pressure gradient is generally positive after $t=\sim 40 \mathrm{~h}$, corresponding with the slight increases of the $2-\mathrm{km}$ convergence and the $2-\mathrm{km}$ vorticity prior to $50-\mathrm{m}$ vorticity (Fig. 12e). After about $t=78 \mathrm{~h}$, the positive pressure gradient near the surface induces convergence there, contributing to the significant increase of 50-m vorticity. In EXP_WS, the $50-\mathrm{m}$ pressure gradient becomes positive, and the vorticity starts to increase at $t=\sim 55 \mathrm{~h}$, whereas the $2-\mathrm{km}$ vorticity has already increased, and positive pressure gradient above the boundary layer has emerged for at least $20 \mathrm{~h}$. Again, after $t=\sim 55 \mathrm{~h}$, the low-level vorticity through the whole boundary layer increases significantly by the effect of convergence. According to the sequential evolution of the vorticity above the boundary layer, the pressure gradient, and the vorticity within the boundary layer in three experiments, one can infer that the aforementioned boundary layer spinup mechanisms in TC intensification can be used in TC formation interpretation as well.

\section{b. Vorticity and divergence budget}

To further investigate how the vorticity amplification and the spinup of the vortex near the surface happens, vorticity and divergence budgets around the occurrence of TC genesis in the three experiments are diagnosed using 6-min model output. In the divergence budget [Eq. (2)], the changes of the PG, the DHF, and the RE usually offset each other. Thus, only the sum (denoted as S1) of these three terms is considered in this study. Negative S1 represents that the PG contributes to convergence, although it should be remembered that this term is actually a gradient imbalance term, in which a negative value indicates that PG is larger than the offsetting RE and DHF terms. Additionally, terms VAD and EVS in Eq. (2) offset each other, and the sum of them is presented by S2 for simplicity in Fig. 13. Terms HAD and SD also offset each other, and the sum of them is denoted as $\mathrm{S} 3$.

According to the vorticity budget, the vorticity tendency at $50 \mathrm{~m}$ becomes basically positive after about $t=$ $92 \mathrm{~h}$ in CTL (Fig. 13a), indicating the significant amplification of the near-surface vorticity. The convergence term dominates the enhancement of vorticity. The friction term near the surface, including turbulence mixing and numerical dissipation, partly damps the effect of the convergence of vorticity. The tilting term is rather small near the surface. According to the divergence budget (Fig. 13b), the convergence tendency (negative values) after $t=88 \mathrm{~h}$ is mainly induced by the pressure gradient term (negative S1), which results from the rapid systemscale surface pressure falling (Fig. 12a) and the gradient wind imbalance. Though the convergence tendency and the term S1 do not monotonically decrease to become continuously negative, they indicate the establishment of a low pressure vortex near the surface (Fig. 13b).

The low-level vorticity amplification mechanism in EXP_CP is analogous to that in CTL, except that the obvious increase of vorticity starts later (about $t=106 \mathrm{~h}$ ) 

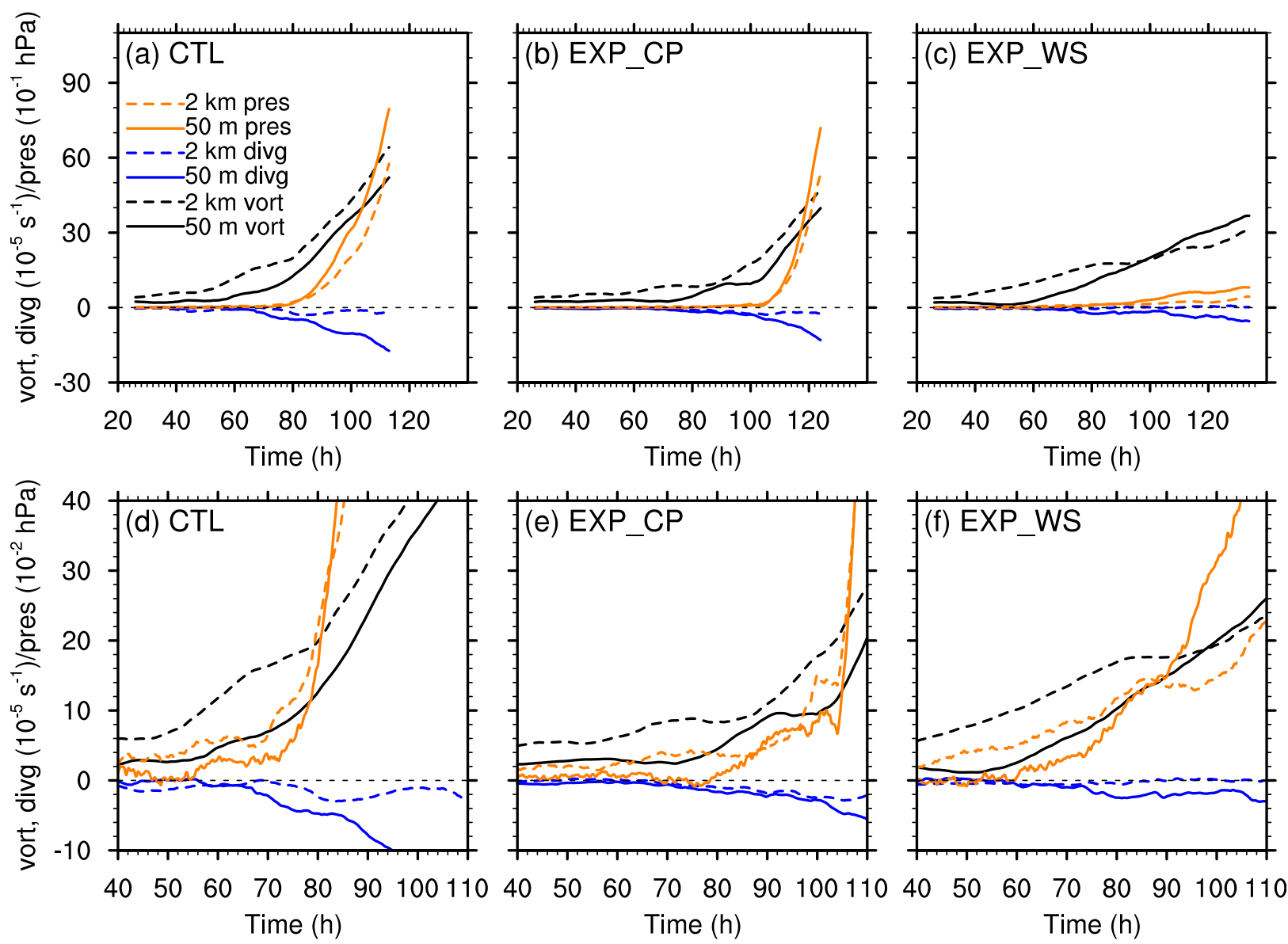

FIG. 12. Time series of 12-h running-mean and area-mean relative vorticity $\left(10^{-5} \mathrm{~s}^{-1}\right)$ at $50 \mathrm{~m}$ (black solid line) and $2 \mathrm{~km}$ (black dashed line), divergence $\left(10^{-5} \mathrm{~s}^{-1}\right)$ at $50 \mathrm{~m}$ (blue solid line) and $2 \mathrm{~km}$ (blue dashed line) $\mathrm{km}$ in a $102 \mathrm{~km} \times 102 \mathrm{~km}$ box centered on the vortex at $1 \mathrm{~km}$, the 12-h running-mean difference between the area-mean 50-m (orange solid line) and 2-km (orange dashed line) pressure [(a)(c) $10^{-1}$ and (d)-(f) $10^{-2} \mathrm{hPa}$ ] in the ring region with a radius of $34-51 \mathrm{~km}$ and within the radius of $17 \mathrm{~km}$ centered on the vortex at $1 \mathrm{~km}$ in (a),(d) CTL, (b),(e) EXP_CP, and (c),(f) EXP_WS. The enlarged figures during early period of vorticity amplification in (a)-(c) are presented in (d)-(f). The horizontal thin dashed line denotes 0 .

in EXP_CP (Figs. 12b, 13c). Such lateness is associated with the postponed intensification of low-level convection and updrafts. The initial increase of $2-\mathrm{km}$ vorticity is also prior to 50-m vorticity (Figs. 12b,e). Similar to CTL, the convergence tendency oscillates rapidly, but term $\mathrm{S} 1$ at $50 \mathrm{~m}$ gradually becomes negative after $t=113 \mathrm{~h}$ (Fig. 13d). Note that there is vorticity convergence during $t=105-108 \mathrm{~h}$ (Fig. 13c), whereas the pressure gradient term (term S1) seems not to be the major contributor to the convergence tendency (Fig. 13d), which is probably related to the small size of the vortex embryo in EXP_CP. One strong convective cell develops first with a low pressure center (Fig. 14c), but a mesoscale low pressure vortex has not formed yet at $t=$ $107 \mathrm{~h}$ in EXP_CP. At $t=110 \mathrm{~h}$, as the mesoscale low pressure system generally strengthens and there is strong convergence into the pressure center (Fig. 14d), the pressure gradient term becomes one of the dominant terms of convergence tendency. As for CTL, the initial vortex with mesoscale low pressure field is larger than that in EXP_CP (Figs. 14a,b). Thus, though the convergence is weak, it is mainly contributed by the pressure gradient term (Figs. 13a,b). The horizontal cross sections of the near-surface virtual temperature perturbations show that the reduction of evaporative cooling produces smaller and weaker patches of cold pools [Figs. 15b(1)-b(3)]. According to the shear-cold pool dynamics, weaker cold pools leads to fewer lowertropospheric updrafts being triggered at the edges. Hence, the updrafts are less organized, compared with CTL [Figs. 15a(3),a(4) and 15b(2),b(3)]. The initial vortex embryo in EXP_CP evolves from one strong convective cell, resembling the evolution in Nolan (2007), instead of through convective cells organizing at 

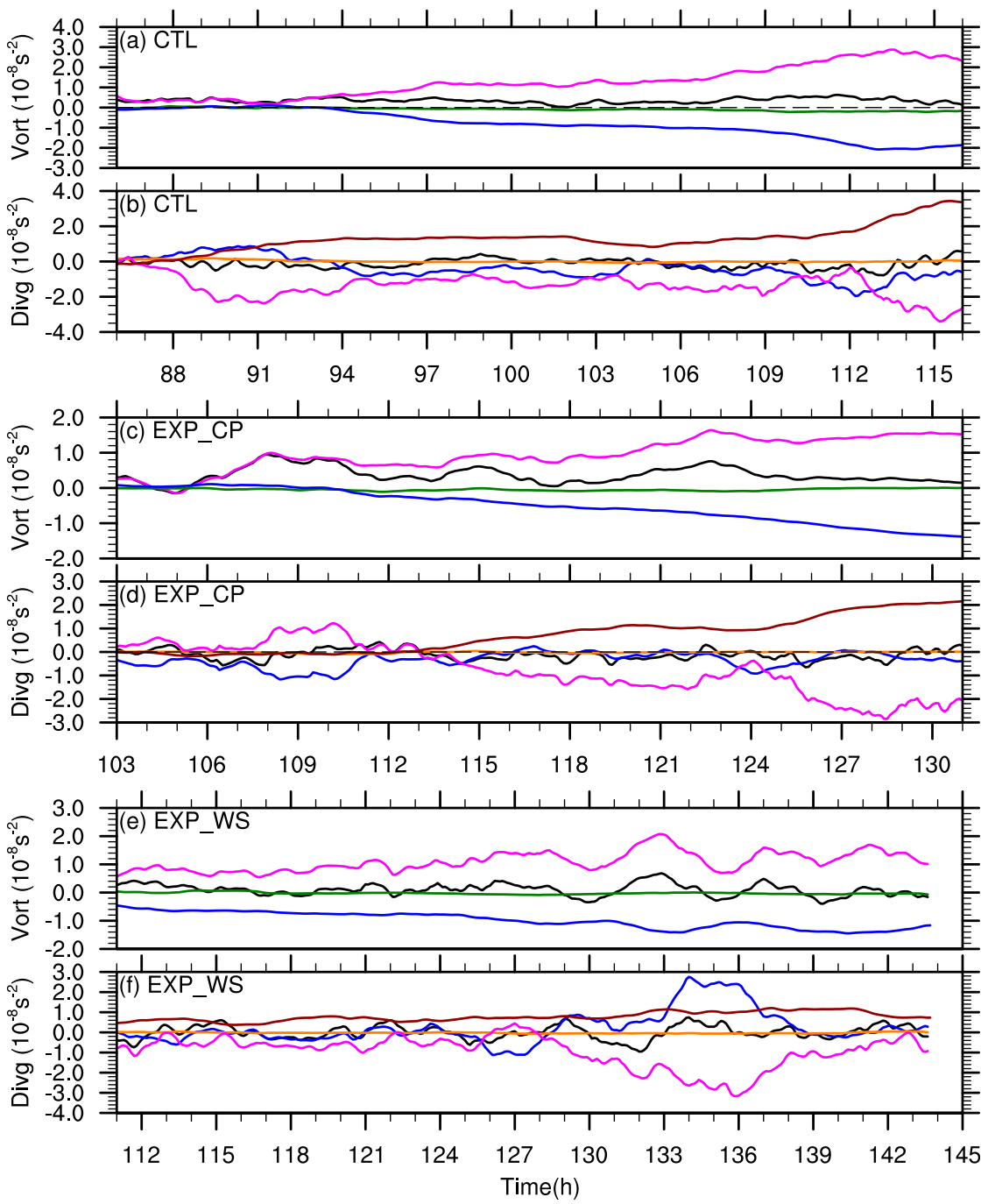

FIG. 13. Time series of the 3-h running-mean and area-mean (a),(c),(e) vorticity budget terms $\left(10^{-8} \mathrm{~s}^{-2}\right)$ and (b),(d),(f) divergence budget terms $\left(10^{-8} \mathrm{~s}^{-2}\right)$ at $50-\mathrm{m}$ altitude in a $102 \mathrm{~km} \times$ $102 \mathrm{~km}$ box centered on the vortex at $1 \mathrm{~km}$ in (a),(b) CTL, (c),(d) EXP_CP, and (e),(f) EXP_WS during TC genesis period: (a),(b) 86-116, (c),(d) 103-131, and (e),(f) 111-145 h. The vorticity budget includes the relative vorticity tendency $(\partial C / \partial t$; black line), the convergence of the horizontal advective flux of vertical vorticity (magenta line), tilting term (green line), and the friction term (blue line). The divergence budget includes the divergence tendency $(\partial \delta / \partial t$; black line), the sum of the change of the gradient of isobaric-layer term, the deformation term, and the rotation effect (term S1; magenta line), the sum of the vertical advection of divergence term and the effect of vertical shear of horizontal wind (term S2; orange line), the sum of the horizontal advection of divergence term and the square of divergence term (term S3; blue line), and the friction term (brown line). Different scales are applied in different panels.

the edges of cold pools as CTL. The small vortex appears representative based on the result of an ensemble of simulations and it is consistent with the absence of a mesoscale updraft forcing mechanism like cold pools. We hypothesize that this leads to the smaller size of the low pressure region in EXP_CP.

In EXP_WS, the vorticity tendency is generally positive with small magnitude before $t=130 \mathrm{~h}$, and then becomes negative occasionally (Fig. 13e), resulting in early but steady vorticity increasing near the surface (Fig. 12c). Consequently, the low pressure vortex in EXP_WS does not intensify as fast as those in CTL and EXP_CP in terms of thr moderate increase of the pressure gradient and areamean convergence (Figs. 12c, 13f). As analyzed above, this phenomenon is partly because the development of convection is interrupted by dry air at the lower troposphere, 

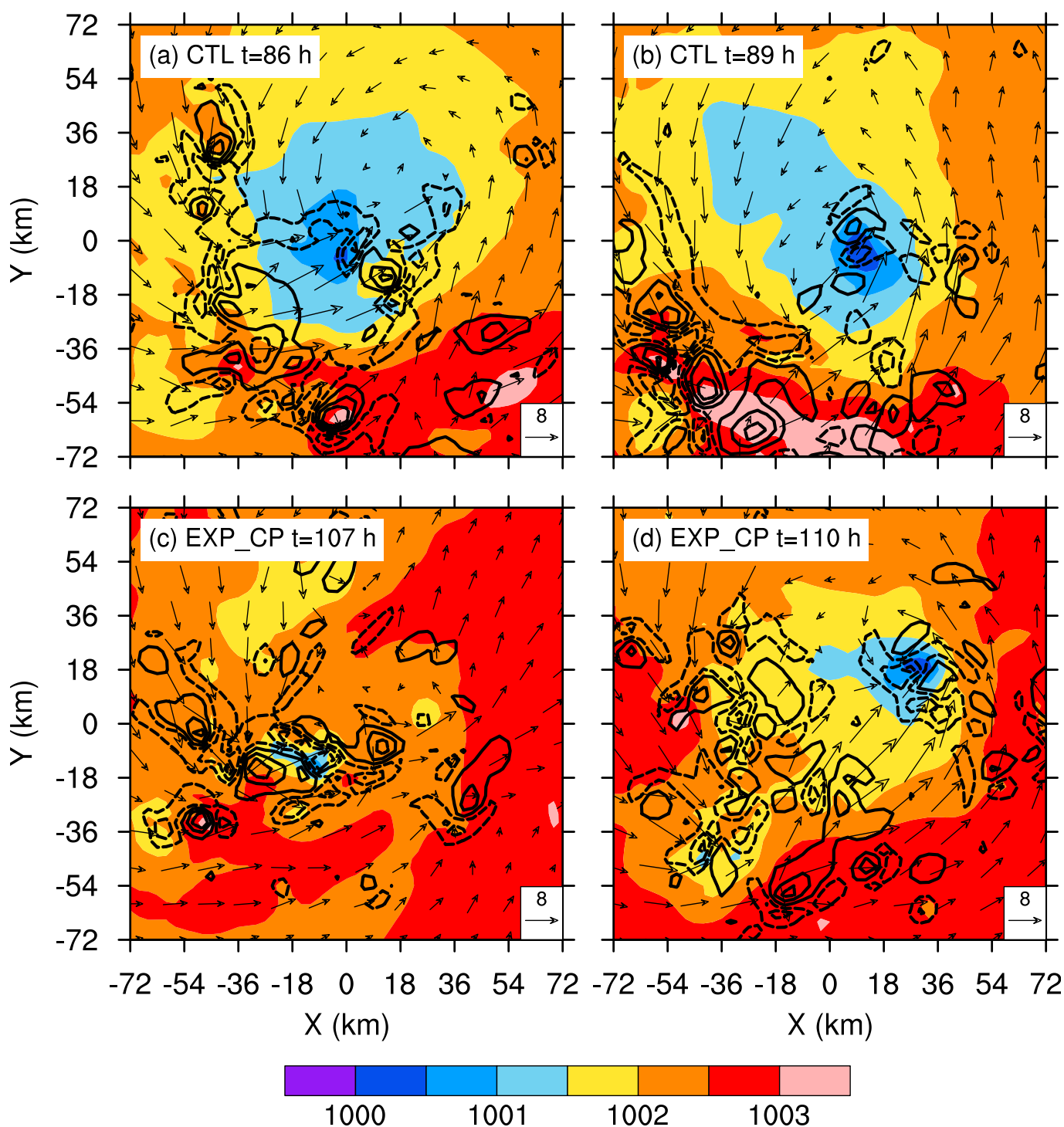

FIG. 14. Horizontal cross sections of convergence (dashed contour) and divergence (solid contour; $10^{-3} \mathrm{~s}^{-1}$ ), pressure (shaded; $\mathrm{hPa}$ ) and wind field (vectors; $\mathrm{m} \mathrm{s}^{-1}$ ) at 50-m altitude at (a) 86 and (b) $89 \mathrm{~h}$ from CTL and at (c) 107 and (d) $110 \mathrm{~h}$ from EXP_CP. The contours represent values from $-2.5 \times 10^{-2}$ to $2.5 \times 10^{-3} \mathrm{~s}^{-1}$ with an interval of $1.0 \times 10^{-3} \mathrm{~s}^{-1}$. The domain follows the vortex at $1-\mathrm{km}$ altitude.

even though a surface vortex has formed, and by the spatial displacement of the vorticity centers at middle and low levels. Besides, affected by the vertical wind shear, the pattern of cold pools and the associated updrafts remain highly asymmetric [Figs. 15c(1)-c(4)]. All these factors inhibit the establishment of the TC's vertically coherent structure and its rapid intensification in EXP_WS.

\section{c. Role of the boundary layer}

In the boundary layer, the convergence/radial inflow caused by the gradient imbalance with the existence of friction is an important contributor to the vorticity amplification and the vortex spinup. While we have shown that the pressure gradient induced in the boundary layer leads to convergence, we more directly explore the role of surface friction, and the essential role of the boundary layer, by conducting additional sensitivity experiments with an altered drag coefficient $C_{d}$, which represents the vertical turbulent momentum flux near the surface. The configurations for these sensitivity experiments are identical to CTL, except that in EXP_LCd, $C_{d}$ is enlarged by a factor of 3, and in EXP_SCd, $C_{d}$ is reduced to $1 / 3$ of its value in CTL. However, because of the special model setup in CTL, the surface entropy exchange coefficients $C_{k}$ would also be changed, increasing with enlarged $\mathrm{C}_{d}$ and decreasing with reduced $C_{d}$. 

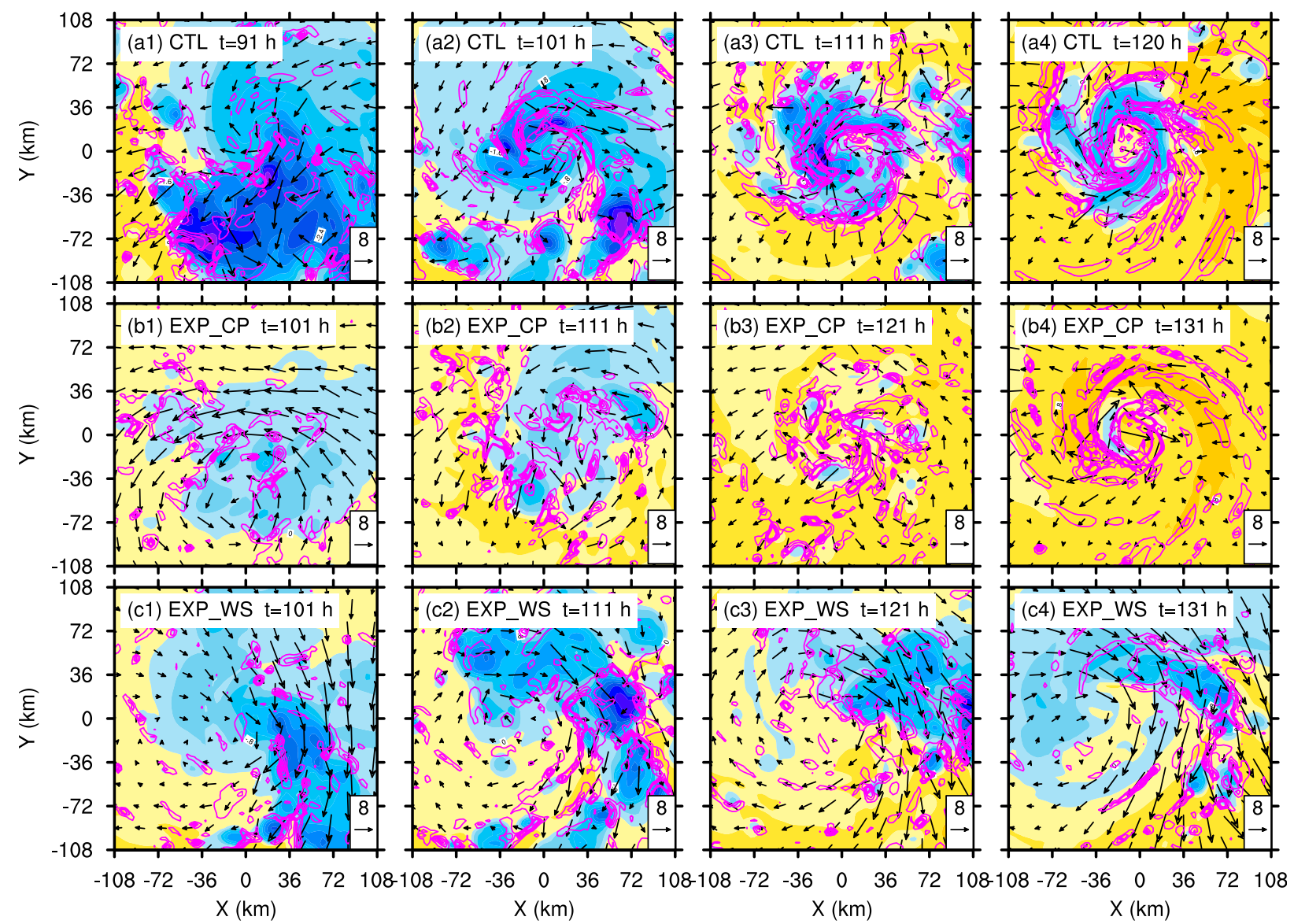

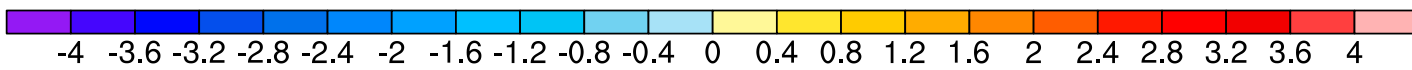

FIG. 15. Horizontal cross sections of virtual temperature anomaly (shaded; K) at $50 \mathrm{~m}$ above the surface, vertical velocity (contours; $\mathrm{m} \mathrm{s}^{-1}$ ) at 500-m altitude, and vertical wind shear (vectors; $\mathrm{m} \mathrm{s}^{-1}$ ) between 6-km and 500-m altitudes at $[\mathrm{a}(1)]$ 91, [a(2)] 101, [a(3)] 111, and [a(4)] $120 \mathrm{~h}$ in CTL; [b(1)] 101, [b(2)] 111, [b(3)] 121, and [b(4)] $131 \mathrm{~h}$ in EXP_CP; and [c(1)] 101, [c(2)] 111, [c(3)] 121, and [c(4)] 131 h in EXP_WS.

Thus, based on the configuration of CTL with $C_{d} \approx$ $1.0 \times 10^{-3}$ and $C_{k}$ from $1.2 \times 10^{-3}$ to $1.4 \times 10^{-3}$, three more sensitivity experiments with fixed $C_{k}\left(1.2 \times 10^{-3}\right)$ and different $C_{d}\left(1.0 \times 10^{-3}, 3.0 \times 10^{-3}, 0.33 \times 10^{-3}\right)$ are conducted. Here, a different surface-layer scheme, wherein $C_{k}$ and $C_{d}$ are specified and remaining fixed over time, is used in this case, without changing the evolution of CTL very much.

Results with larger $C_{d}$ and $C_{k}$ indicate that a TC forms earlier than that in CTL and with a higher intensification rate, whereas with reduced $C_{d}$ and $C_{k}$, the TC's formation is delayed for about $50 \mathrm{~h}$ and then it develops rather slowly (Figs. 16a,b), despite the existence of a nearly saturated air column. When $C_{k}$ is fixed, the intensification rate is also proportional to $C_{d}$ (Fig. 17), indicating that the changes in $C_{d}$ have a dominant effect on the intensification rate variation. These results are consistent with the previous studies (e.g., Montgomery et al. 2010;
Kilroy et al. 2017b; Peng et al. 2018), suggesting that the intensification rate of the vortex was found to increase with increasing surface drag coefficient. Similar diagnoses for the sequential evolution of the low-level vorticity and convergence are presented in Figs. 16c-f. Following a steady increase of $2-\mathrm{km}$ vorticity, the positive pressure gradient and continuous convergence in the boundary layer occur after $t=\sim 44 \mathrm{~h}$ in EXP LCd. After that time, the near-surface vorticity starts to increase remarkably. As for EXP_SCd, the pressure gradient becomes generally positive at $t=\sim 97 \mathrm{~h}$, corresponding with convergence near the surface. At the same time, the $50-\mathrm{m}$ vorticity starts to increase. However, the magnitudes of the pressure gradient and the convergence are much weaker than those in EXP_LCd. The results suggest that even though friction in the boundary layer reduces the near-surface wind speed everywhere, it induces an imbalance of forces in the 

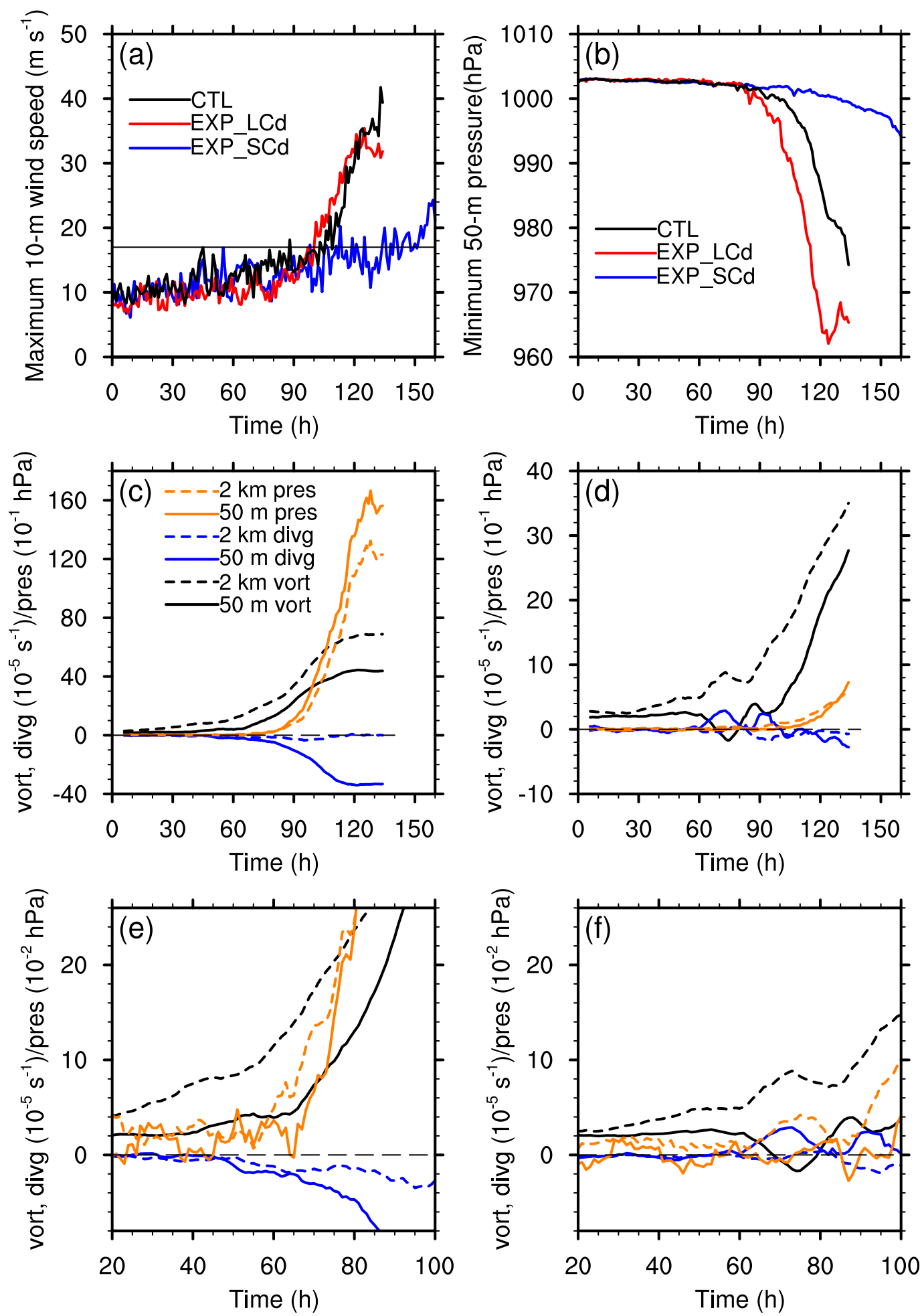

FIG. 16. Time series of (a) maximum $10-\mathrm{m}$ wind speed $\left(\mathrm{m} \mathrm{s}^{-1}\right)$ and (b) minimum $50-\mathrm{m}$ pressure (hPa) in CTL (thick black line), EXP_LCd (thick red line), and EXP_SCd (thick blue line). The horizontal solid line in (a) denotes $17 \mathrm{~m} \mathrm{~s}^{-1}$, which is the minimum wind speed of a tropical storm. (c)-(f) As in Fig. 12, but for (c),(e) EXP_LCd and (d),(f) EXP_SCd. 

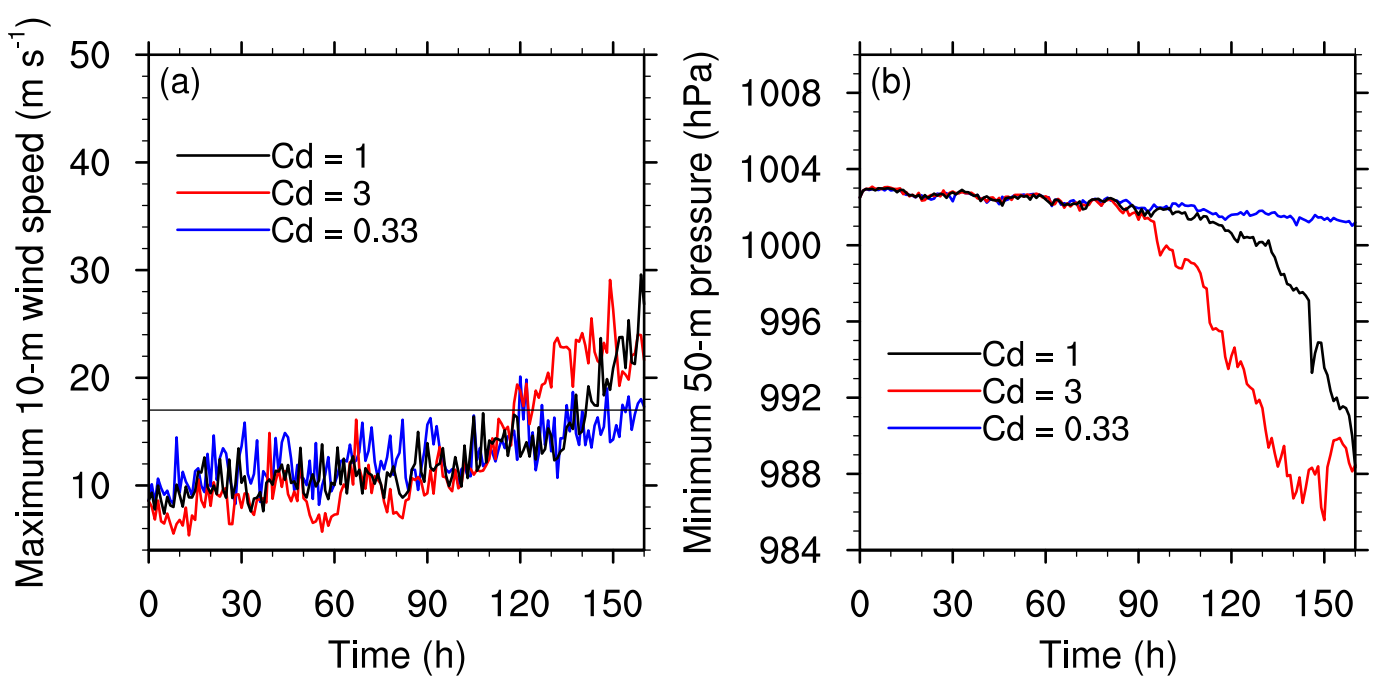

FIG. 17. Time series of (a) maximum 10-m wind speed $\left(\mathrm{m} \mathrm{s}^{-1}\right)$ and (b) minimum $50-\mathrm{m}$ pressure $(\mathrm{hPa})$ from simulations with different drag coefficients $\left(C_{d}=1.0 \times 10^{-3}, 3.0 \times 10^{-3}, 0.33 \times 10^{-3}\right)$ and a fixed surface entropy exchange coefficient $\left(C_{k}=1.2 \times 10^{-3}\right)$.

radial direction when there is a pressure gradient imposed through the depth of the boundary layer. The resultant frictional convergence associated with the pressure gradient promotes the process of vortex spinup in TC genesis from the top of the boundary layer to the near surface. The spinup role of the frictional boundary layer is more important than its direct reduction of wind speed in TC formation.

\section{Summary and conclusions}

In this study, the effect of thermodynamic changes on a surface vortex spinup with a preexisting midtropospheric vortex is explored utilizing an idealized model. Sensitivity experiments are conducted by nearly eliminating evaporative cooling below cloud base to weaken cold pools and by adding weak vertical wind shear. Sensitivity and control runs all show that as lowertropospheric air approaches saturation before TC genesis, lower-tropospheric convective updrafts become stronger and more numerous. This increases the vertical gradient of vertical mass flux beneath $2 \mathrm{~km}$ and thus leads to greater horizontal convergence and a spinup of vorticity beneath $2 \mathrm{~km}$. Here, the vertical gradient of mass flux, rather than the shape of mass flux profile, is emphasized. Our study shows that the mass flux profiles are not technically bottom heavy during TC genesis, similar as the results in Kilroy et al. (2018) that the height of the maximum mass flux is not systematically lowered prior to the rapid spinup of the low-level circulation.

The delay in near saturation over a deep layer that results from adding vertical wind shear in EXP_WS reduces the number and intensity of updrafts at $2 \mathrm{~km}$ and thus delays the intensification process as well. The addition of weak wind shear induces a misalignment of the mid- and lower-tropospheric vortices that allows a pathway for dry air to enter above the lower-tropospheric vortex or beneath the midtropospheric vortex. Reduced relative humidity generally inhibits convection; strong updrafts are fewer, and updrafts overall are weaker, compared with CTL, thus reducing the upward mass flux and the associated vertical gradient of mass flux in the lower troposphere and leading to a slower amplification of vorticity. The moistening due to convection is thus inhibited and has difficulty overcoming the systemrelative source of dry air.

The near removal of subcloud evaporation cooling drastically reduces the strength of cold pools and, hence, practically eliminates the cold pool-shear mechanism for enhancing lower-tropospheric updrafts in EXP_CP. However, the reduced stratification in the lower troposphere compensates for the absence of an organizing agent such as cold pools. Thus, strong deep convection still occurs. Our study shows that overall, removal of cold pools does not prevent TC genesis, but just delays the genesis for a short time. A comparable intensification rate occurs with cold pools removed, albeit with a smaller near-surface vortex, suggesting that strong surface cold pools and their associated lifting are not essential for genesis.

While the near-surface convergence associated with the sharp gradient in vertical mass flux is consistent with vorticity spinup near the surface, it is not the entire story. We explored the role of the boundary layer 
through a detailed budget of vorticity and divergence at $z=50 \mathrm{~m}$ (the lowest model level) and by varying the drag coefficient. Following an increase of vorticity above the boundary layer, accompanied by a nearly balanced pressure gradient, the near-surface pressure drops accordingly, and the pressure gradient exceeds what is balanced by nascent surface rotation. Near-surface convergence ensues and vorticity increases. In general, the convergence is not steady. Rather, it pulsates with the variability of small-scale convection, consistent with the rotating convective updraft paradigm proposed by Montgomery et al. (2006) and Montgomery and Smith (2014).

Furthermore, we find that the near-surface spinup of vorticity is sensitive to the surface drag coefficient in our control simulation, with spinup inhibited for reduced drag, and hastened slightly for enhanced drag, despite roughly similar vorticity above the boundary layer. This result, combined with the diagnosis of divergence tendency near the surface, implies that the intensification of vertical mass flux in the lower troposphere is necessary, but not sufficient, for genesis. However, for realistic values of surface drag, a strong increase of vorticity above the boundary layer, within a deep column of enhanced vorticity, is still a strong predictor of genesis.

Although the highly idealized simulations conducted herein ignore the synoptic-scale precursors to genesis, the strengthening of the low-level updrafts is similar to the VHT paradigm (Montgomery et al. 2006) and also induces changes in vertical mass flux prior to genesis (Raymond et al. 2011). Besides, the role of the surface frictional drag played in promoting vorticity spinup is similar to the boundary layer spinup mechanism (Smith et al. 2009). Thus, the convection-scale processes and the near-surface vortex evolution process described here should be operating in the real atmosphere. Our study also tries to tell an entire story of TC genesis by associating the VHTs paradigm (Montgomery et al. 2006) with the bottom-heavy mass flux-profile hypothesis (Raymond et al. 2011; Raymond 2012) and the boundary layer mechanism (Smith et al. 2009). Nevertheless, we should note that the $3-\mathrm{km}$ grid scale in these simulations may not be sufficient to resolve all the convection-scale processes as well as the finer structure of cold pools. Though our results should be consistent, further investigation with much finer resolution is needed in future work.

Further work should focus on the role of boundary layer processes coupled with the evolution of vorticity in the lower troposphere to derive a complete view of the genesis process. Though the air column is still near saturated even if the surface drag is reduced, the upward lower-tropospheric vertical mass flux is much weaker than with the control or enhanced surface drag. One factor may be a reduced shallow vertical circulation induced by Ekman-like dynamics. One expects such a vertical circulation when the vorticity at the top of the boundary layer is positive, provided that the boundary layer is in a quasi-steady state. However, Raymond and Herman (2012) caution further that an Ekman-like description of the boundary layer may not be appropriate. Still, our results, consistent with Kilroy et al. (2017b), imply that convection is organized by the boundary layer to some degree and that important influences of friction can occur in the earliest stages of development. Further examination of the causal link between the boundary layer and convective organization is required, particularly when the organization of convection is altered by factors such as vertical wind shear.

Acknowledgments. The authors acknowledge the helpful suggestions of Dr. Richard Rotunno of NCAR, the help of Dr. George Bryan of NCAR in CM1 model modification, and Dr. Lulin Xue of NCAR for providing computing resource. Funding for this research was provided by the National Center for Atmospheric Research through support from the National Science Foundation under Cooperative Agreement AGS-1755088.

\section{REFERENCES}

Abarca, S. F., and M. T. Montgomery, 2013: Essential dynamics of secondary eyewall formation. J. Atmos. Sci., 70, 3216-3230, https://doi.org/10.1175/JAS-D-12-0318.1.

Bell, M. M., and M. T. Montgomery, 2010: Sheared deep vortical convection in pre-depression Hagupit during TCS08. Geophys. Res. Lett., 37, L06802, https://doi.org/10.1029/2009GL042313.

Bister, M., and K. A. Emanuel, 1997: The genesis of Hurricane Guillermo: TEXMEX analyses and a modeling study. Mon. Wea. Rev., 125, 2662-2682, https://doi.org/10.1175/ 1520-0493(1997)125<2662:TGOHGT>2.0.CO;2.

Böing, S. J., H. J. Jonker, A. P. Siebesma, and W. W. Grabowski, 2012: Influence of the subcloud layer on the development of a deep convective ensemble. J. Atmos. Sci., 69, 2682-2698, https://doi.org/10.1175/JAS-D-11-0317.1.

Bryan, G. H., and J. M. Fritsch, 2002: A benchmark simulation for moist nonhydrostatic numerical models. Mon. Wea. Rev., 130, 2917-2928, https://doi.org/10.1175/1520-0493(2002)130<2917: ABSFMN $>2.0 . \mathrm{CO} ; 2$.

__ , and H. Morrison, 2012: Sensitivity of a simulated squall line to horizontal resolution and parameterization of microphysics. Mon. Wea. Rev., 140, 202-225, https://doi.org/ 10.1175/MWR-D-11-00046.1.

Chou, M. D., and M. J. Suarez, 1999: A solar radiation parameterization (CLIRAD-SW) for atmospheric studies. NASA Tech. Memo. NASA/TM-1990-104606, 51 pp.

Davis, C. A., 2015: The formation of moist vortices and tropical cyclones in idealized simulations. J. Atmos. Sci., 72, 34993516, https://doi.org/10.1175/JAS-D-15-0027.1.

, and T. J. Galarneau Jr., 2009: The vertical structure of mesoscale convective vortices. J. Atmos. Sci., 66, 686-704, https:// doi.org/10.1175/2008JAS2819.1. 
Freismuth, T. M., B. Rutherford, M. A. Boothe, and M. T. Montgomery, 2016: Why did the storm ex-Gaston (2010) fail to redevelop during the PREDICT experiment? Atmos. Chem. Phys., 16, 8511-8519, https://doi.org/10.5194/acp-16-8511-2016.

Gjorgjievska, S., and D. J. Raymond, 2014: Interaction between dynamics and thermodynamics during tropical cyclogenesis. Atmos. Chem. Phys., 14, 3065-3082, https://doi.org/10.5194/ acp-14-3065-2014.

Hendricks, E. A., M. T. Montgomery, and C. A. Davis, 2004: The role of "vortical" hot towers in the formation of Tropical Cyclone Diana (1984). J. Atmos. Sci., 61, 1209-1232, https:// doi.org/10.1175/1520-0469(2004)061<1209:TROVHT>2.0.CO;2.

Hong, S. Y., Y. Noh, and J. Dudhia, 2006: A new vertical diffusion package with an explicit treatment of entrainment processes. Mon. Wea. Rev., 134, 2318-2341, https://doi.org/10.1175/MWR3199.1.

Houze, R. A., Jr., W. C. Lee, and M. M. Bell, 2009: Convective contribution to the genesis of Hurricane Ophelia (2005). Mon. Wea. Rev., 137, 2778-2800, https://doi.org/10.1175/2009MWR2727.1.

James, R. P., and P. M. Markowski, 2010: A numerical investigation of the effects of dry air aloft on deep convection. Mon. Wea. Rev., 138, 140-161, https://doi.org/10.1175/ 2009MWR3018.1.

Kilroy, G., and R. K. Smith, 2013: A numerical study of rotating convection during tropical cyclogenesis. Quart. J. Roy. Meteor. Soc., 139, 1255-1269, https://doi.org/10.1002/qj.2022.

—_ _ - and M. T. Montgomery, 2017a: A unified view of tropical cyclogenesis and intensification. Quart. J. Roy. Meteor. Soc., 143, 450-462, https://doi.org/10.1002/qj.2934.

— M. T. Montgomery, and R. K. Smith, 2017b: The role of boundary-layer friction on tropical cyclogenesis and subsequent intensification. Quart. J. Roy. Meteor. Soc., 143, 2524 2536, https://doi.org/10.1002/qj.3104.

— , R. K. Smith, and M. T. Montgomery, 2018: The role of heating and cooling associated with ice processes on tropical cyclogenesis and intensification. Quart. J. Roy. Meteor. Soc., 144, 99-114, https://doi.org/10.1002/qj.3187.

Lewellen, D. C., and W. S. Lewellen, 2007: Near-surface intensification of tornado vortices. J. Atmos. Sci., 64, 2176-2194, https://doi.org/10.1175/JAS3965.1.

Montgomery, M. T., and R. K. Smith, 2014: Paradigms for tropical cyclone intensification. Aust. Meteor. Oceanogr. J., 64, 37-66, https://doi.org/10.22499/2.6401.005.

_ , M. E. Nicholls, T. A. Cram, and A. B. Saunders, 2006: A vortical hot tower route to tropical cyclogenesis. J. Atmos. Sci., 63, 355-386, https://doi.org/10.1175/JAS3604.1.

_ , V. S. Nguyen, J. Persing, and R. K. Smith, 2009: Do tropical cyclones intensify by WISHE? Quart. J. Roy. Meteor. Soc., 135, 1697-1714, https://doi.org/10.1002/qj.459.

_ R. K. Smith, and S. V. Nguyen, 2010: Sensitivity of tropicalcyclone models to the surface drag coefficient. Quart. J. Roy. Meteor. Soc., 136, 1945-1953, https://doi.org/10.1002/qj.702.

Nguyen, S. V., R. K. Smith, and M. T. Montgomery, 2008: Tropical cyclone intensification and predictability in three dimensions. Quart. J. Roy. Meteor. Soc., 134, 563-582, https://doi.org/10.1002/qj.235.

Nolan, D. S., 2007: What is the trigger for tropical cyclogenesis? Aust. Meteor. Mag., 56, 241-266.

Peng, K., R. Rotunno, and G. Bryan, 2018: Evaluation of a timedependent model for the intensification of tropical cyclones. J. Atmos. Sci., 75, 2125-2138, https://doi.org/10.1175/JAS-D17-0382.1.

Persing, J., M. T. Montgomery, J. McWilliams, and R. K. Smith, 2013: Asymmetric and axisymmetric dynamics of tropical cyclones. Atmos. Chem. Phys., 13, 12299-12341, https:// doi.org/10.5194/acp-13-12299-2013.

Raymond, D. J., 2012: Balanced thermal structure of an intensifying tropical cyclone. Tellus, 64A, 19181, https://doi.org/ 10.3402/tellusa.v64i0.19181.

— tropical cyclogenesis. Geophys. Res. Lett., 34, L06811, https:// doi.org/10.1029/2006GL028607.

, and C. López-Carrillo, 2011: The vorticity budget of developing Typhoon Nuri (2008). Atmos. Chem. Phys., 11, 147163, https://doi.org/10.5194/acp-11-147-2011.

, and M. J. Herman, 2012: Frictional convergence, atmospheric convection, and causality. Atmósfera, 25, 253-267.

_ S. L. Sessions, and C. López-Carrillo, 2011: Thermodynamics of tropical cyclogenesis in the Northwest Pacific. J. Geophys. Res., 116, D18101, https://doi.org/10.1029/2011JD015624.

Reasor, P. D., M. T. Montgomery, and L. F. Bosart, 2005: Mesoscale observations of the genesis of Hurricane Dolly (1996). J. Atmos. Sci., 62, 3151-3171, https://doi.org/10.1175/JAS3540.1.

Ritchie, E. A., and G. J. Holland, 1997: Scale interactions during the formation of Typhoon Irving. Mon. Wea. Rev., 125, 1377-1396, https:// doi.org/10.1175/1520-0493(1997)125<1377:SIDTFO > 2.0.CO;2.

Sanger, N. T., M. T. Montgomery, R. K. Smith, and M. M. Bell, 2014: An observational study of tropical cyclone spinup in Supertyphoon Jangmi (2008) from 24 to 27 September. Mon. Wea. Rev., 142, 3-28, https://doi.org/10.1175/MWR-D-12-00306.1.

Schmidt, C., and R. K. Smith, 2016: Tropical cyclone evolution in a minimal axisymmetric model revisited. Quart. J. Roy. Meteor. Soc., 142, 1505-1516, https://doi.org/10.1002/qj.2753.

Simpson, J., E. Ritchie, G. J. Holland, J. Halverson, and S. Stewart, 1997: Mesoscale interactions in tropical cyclone genesis. Mon. Wea. Rev., 125, 2643-2661, https://doi.org/10.1175/15200493(1997)125<2643:MIITCG > 2.0.CO;2.

Sippel, J. A., J. W. Nielsen-Gammon, and S. E. Allen, 2006: The multiple-vortex nature of tropical cyclogenesis. Mon. Wea. Rev., 134, 1796-1814, https://doi.org/10.1175/MWR3165.1.

Smith, R. K., and M. T. Montgomery, 2012: Observations of the convective environment in developing and non-developing tropical disturbances. Quart. J. Roy. Meteor. Soc., 138, 17211739, https://doi.org/10.1002/qj.1910.

$\longrightarrow,-$, and V. S. Nguyen, 2009: Tropical cyclone spin-up revisited. Quart. J. Roy. Meteor. Soc., 135, 1321-1335, https://doi.org/ 10.1002/qj.428.

Wang, Y., Y. Huang, and X. Cui, 2018: Impact of mid- and upperlevel dry air on tropical cyclone genesis and intensification: A modeling study of Durian (2001). Adv. Atmos. Sci., 35, 15051521, https://doi.org/10.1007/s00376-018-8039-0.

Wang, Z., 2012: Thermodynamic aspects of tropical cyclone formation. J. Atmos. Sci., 69, 2433-2451, https://doi.org/10.1175/JAS-D-11-0298.1.

Zawislak, J., and E. J. Zipser, 2014: Analysis of the thermodynamic properties of developing and nondeveloping tropical disturbances using a comprehensive dropsonde dataset. Mon. Wea. Rev., 142, 1250-1264, https://doi.org/10.1175/MWR-D-13-00253.1.

Zhang, D. L., Y. Liu, and M. K. Yau, 2001: A multiscale numerical study of Hurricane Andrew (1992). Part IV: Unbalanced flows. Mon. Wea. Rev., 129, 92-107, https://doi.org/10.1175/ 1520-0493(2001)129<0092:AMNSOH > 2.0.CO;2.

Zhang, J. A., and F. D. Marks, 2015: Effects of horizontal diffusion on tropical cyclone intensity change and structure in idealized three-dimensional numerical simulations. Mon. Wea. Rev., 143, 3981-3995, https://doi.org/10.1175/MWR-D-14-00341.1. 